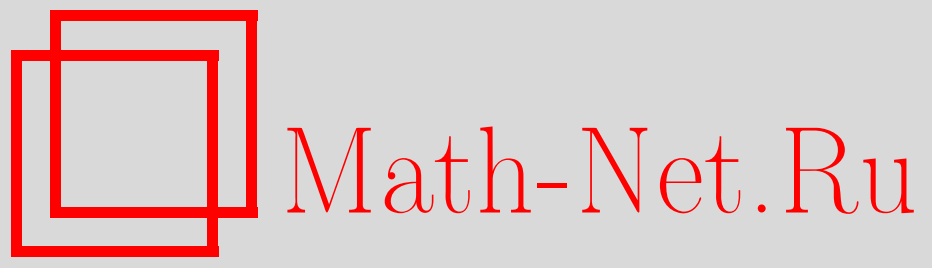

М. В. Миронюк, Г. М. Фельдман, Независимые линейные статистики на цилиндрах, Теория вероятн. и ее примен., 2014, том 59, выпуск 2, 252-275

DOI: https://doi.org/10.4213/tvp4565

Использование Общероссийского математического портала Math-Net.Ru подразумевает, что вы прочитали и согласны с пользовательским соглашением http://www.mathnet.ru/rus/agreement

Параметры загрузки:

IP : 54.198 .187 .58

26 апреля 2023 г., 13:47:01

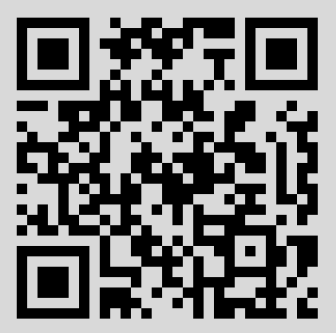




\section{НЕЗАВИСИМЫЕ ЛИНЕЙНЫЕ СТАТИСТИКИ НА ЦИЛИНДРАХ}

Пусть либо $X=\mathbf{R} \times \mathbf{T}$, либо $X=\Sigma_{\boldsymbol{a}} \times \mathbf{T}$, где $\mathbf{R}-$ группа вещественных чисел, $\mathbf{T}$ - группа вращений окружности, $\Sigma_{\boldsymbol{a}}$ $\boldsymbol{m}$-адический соленоид. Пусть $\alpha_{i j}, i, j=1,2,3,-$ топологические автоморфизмы группы $X$. Доказан следующий аналог теоремы Скитовича-Дармуа для группы $X$. Пусть $\xi_{j}, j=1,2,3$, независимые случайные величины со значениями в группе $X$ и с распределениями $\mu_{j}$ такими, что их характеристические функции не обращаются в нуль. Если линейные статистики $L_{1}=\alpha_{11} \xi_{1}+$ $\alpha_{12} \xi_{2}+\alpha_{13} \xi_{3}, L_{2}=\alpha_{21} \xi_{1}+\alpha_{22} \xi_{2}+\alpha_{23} \xi_{3}, L_{3}=\alpha_{31} \xi_{1}+\alpha_{32} \xi_{2}+\alpha_{33} \xi_{3}$ независимы, то все $\mu_{j}$ - гауссовские распределения.

Ключевые слова и фразы: независимые линейные статистики, гауссовское распределение, локально компактная абелева группа.

1. Введение. В.П. Скитовичем и Ж. Дармуа была доказана следующая теорема, характеризующая гауссовские распределения на вещественной прямой.

Теорема A ([1], [2], см. также [3, гл. 3]). Пусть $\xi_{j}, j=1,2, \ldots, n$, $n \geqslant 2$, - независимье случайнье величинь и $\alpha_{j}, \beta_{j}$ - ненулевые константыл. Если линейнье статистики $L_{1}=\alpha_{1} \xi_{1}+\cdots+\alpha_{n} \xi_{n} u L_{2}=$ $\beta_{1} \xi_{1}+\cdots+\beta_{n} \xi_{n}$ независимьи, то случайнье величинь $\xi_{j}-$ гауссовские.

Групповым аналогам теоремы Скитовича-Дармуа посвящено большое число исследований (см., например, [4]-[15], а также [16], где можно найти дополнительные ссылки). В частности, в [11] была доказана следующая теорема.

Теорема В. Пусть $X-$ локально компактная абелева группа, удовлетворяющая второй аксиоме счетности. Предположим, что группа $X$ не содержит подгруппь, топологически изоморфной группе вращений окружности. Пусть $\xi_{j}, j=1,2, \ldots, n, n \geqslant 2$, - независимье случайнье величинь со значениями в $X$, имеющие не обращающиеся в нуль характеристические функиии. Пусть $\alpha_{j}, \beta_{j}-$ топологические автоморфизмы группь $X$. Если линейнье статистики $L_{1}=\alpha_{1} \xi_{1}+\cdots+\alpha_{n} \xi_{n} u L_{2}=\beta_{1} \xi_{1}+\cdots+\beta_{n} \xi_{n}$ независимы, то случайнье величинь $\xi_{j}-$ гауссовские.

*Физико-технический институт низких температур им. Б. И. Веркина НАН Украины, Харьков, Украина; e-mail: myronyuk@ilt.kharkov.ua; feldman@ilt.kharkov.ua 
Пусть $\mathbf{T}$ обозначает группу вращений окружности (одномерный тор), т.е. $\mathbf{T}=\{z \in \mathbf{C}:|z|=1\}$. Как было замечено в [17], теорема В неверна на группе $\mathbf{T}$ уже для двух линейных статистик $L_{1}=\xi_{1}+\xi_{2}$ и $L_{2}=\xi_{1}-\xi_{2}$. Отсюда следует, что если группа $X$ содержит подгруппу, топологически изоморфную $\mathbf{T}$, то класс распределений, характеризующийся независимостью линейных статистик $L_{1}=\alpha_{1} \xi_{1}+\alpha_{2} \xi_{2}$ и $L_{2}=\beta_{1} \xi_{1}+\beta_{2} \xi_{2}$, вообще говоря, содержит не только гауссовские распределения. Другими словами, для групп, содержащих подгруппу, топологически изоморфную $\mathbf{T}$, теорема Скитовича-Дармуа неверна, если рассматривать две линейные статистики от двух независимых случайных величин.

Цель настоящей статьи состоит в доказательстве следующего достаточно неожиданного результата. Мы докажем, что для некоторых локально компактных абелевых групп, содержащих подгруппу, топологически изоморфную $\mathbf{T}$, теорема Скитовича-Дармуа верна, если рассматривать три линейные статистики от трех независимых случайных величин.

Рассмотрим следующую общую задачу.

Задача 1. Пусть $X$ - локально компактная абелева группа, удовлетворяющая второй аксиоме счетности. Пусть $\xi_{j}, j=1,2, \ldots, n$, $n \geqslant 2$, - независимье случайнье величинь со значениями в группе $X$ и с распределениями $\mu_{j}$ такими, что их характеристические функции не обращаются в нуль. Пусть $\alpha_{i j}, i, j=1,2, \ldots, n,-$ топологические автоморфизмы группь $X$. Предположим, что линейнье статистики $L_{i}=\alpha_{i 1} \xi_{1}+\cdots+\alpha_{i n} \xi_{n}, i=1,2, \ldots, n$, независимьл. Oписать распределения $\mu_{j}$.

Как следует из теоремы В, если выполнены условия задачи 1 и группа $X$ не содержит подгруппы, топологически изоморфной $\mathbf{T}$, то $\mu_{j}-$ гауссовские распределения. Поэтому содержательная часть задачи 1 относится к случаю, когда группа $X$ содержит подгруппу, топологически изоморфную $\mathbf{T}$.

Отметим, что, как доказано в [11], решение задачи 1 может быть сведено к ее решению для связной локально компактной абелевой группы, одной из характеристик которой является ее размерность. Если размерность группы $X$ равна 1 и группа $X$ содержит подгруппу, топологически изоморфную $\mathbf{T}$, то $X \cong \mathbf{T}$. Как доказано в [18], в условиях задачи 1 при $n=2$ распределения $\mu_{j}$ являются либо гауссовскими, либо свертками гауссовских распределений с зарядами, сосредоточенными на подгруппе, порожденной элементом порядка 2.

Предположим теперь, что размерность группы $X$ равна 2 и группа $X$ содержит подгруппу, топологически изоморфную T. Тогда группа $X$ топологически изоморфна одной из следующих групп: $\mathbf{R} \times \mathbf{T}$, где $\mathbf{R}-$ группа вещественных чисел, $\Sigma_{\boldsymbol{a}} \times \mathbf{T}$, где $\Sigma_{\boldsymbol{a}}-\boldsymbol{a}$-адический 
соленоид, и $\mathbf{T}^{2}$. Для групп $\mathbf{R} \times \mathbf{T}$ и $\Sigma_{\boldsymbol{a}} \times \mathbf{T}$ при $n=2$ задача 1 была решена в работе [15]. Оказалось, что, так же как и в случае $X=\mathbf{T}$, распределения $\mu_{j}$ являются либо гауссовскими, либо свертками гауссовских распределений с зарядами, сосредоточенными на подгруппе, порожденной элементом порядка 2 в подгруппе $\mathbf{T}$. Для группы $\mathbf{T}^{2}$ при $n=2$ полностью решить задачу 1 не удалось, но удалось описать все топологические автоморфизмы $\alpha_{j}, \beta_{j}$ группы $\mathbf{T}^{2}$ такие, что из независимости линейных статистик $L_{1}=\alpha_{1} \xi_{1}+\alpha_{2} \xi_{2}$ и $L_{2}=\beta_{1} \xi_{1}+\beta_{2} \xi_{2}$ следует, что распределения $\mu_{j}$ - гауссовские [12].

Насколько нам известно, задача 1 при $n>2$ ранее не изучалась. Более того, во всех предыдущих работах, посвященных теореме Скитовича-Дармуа на группах, рассматривались две линейные статистики от $n \geqslant 2$ независимых случайных величин. Но в групповой ситуации, в отличие от классической, рассмотрение двух линейных статистик от $n>2$ независимых случайных величин, вообще говоря, не дает возможности характеризовать гауссовские распределения или их аналоги (см., например, $[16, \S 14]$ ). Как вытекает из доказанных в статье теорем 1 и 2 , ситуация меняется, если рассматривать $n$ линейных статистик от $n$ независимых случайных величин.

В настоящей статье мы полностью решаем задачу 1 в случае $n=3$ для группы $\mathbf{R} \times \mathbf{T}$, а затем, опираясь на полученный результат, и для группы $\Sigma_{\boldsymbol{a}} \times \mathbf{T}$. Ответ оказывается весьма неожиданным: распределения $\mu_{j}$ должны быть гауссовскими. Другими словами, если мы будем рассматривать три линейные статистики от трех независимых случайных величин, то для указанных групп снова верна теорема СкитовичаДармуа. Подчеркнем, что, как отмечено выше, если рассматривать две линейные статистики от двух независимых случайных величин, то для указанных групп распределения $\mu_{j}$ могут быть как гауссовскими, так и свертками гауссовских распределений с зарядами, т.е. в этом случае теорема Скитовича-Дармуа неверна. Кроме того, мы видим, что на таких группах, в отличие от классической ситуации, класс распределений, характеризующийся независимостью $n$ линейных статистик от $n$ независимых случайных величин, зависит от $n$.

\section{2. Обозначения и определения. Формулировка основной те-} оремы. Пусть $X$ - локально компактная абелева группа, удовлетворяющая второй аксиоме счетности, $Y$ - ее группа характеров, $(x, y)$ значение характера $y \in Y$ на элементе $x \in X$, Aut $(X)$ - группа топологических автоморфизмов $X$. Если $H$ - подгруппа группы $Y$, то обозначим через $A(X, H)=\{x \in X:(x, y)=1$ для любого $y \in H\}$ ее аннулятор. Если $X_{1}$ и $X_{2}$ - локально компактные абелевы группы, то для любого непрерывного гомоморфизма $\alpha: X_{1} \mapsto X_{2}$ определим сопряженный гомоморфизм $\widetilde{\alpha}: Y_{2} \mapsto Y_{1}$ формулой $\left(x_{1}, \widetilde{\alpha} y_{2}\right)=\left(\alpha x_{1}, y_{2}\right)$ для всех 
$x_{1} \in X_{1}, y_{2} \in Y_{2}$. Обозначим через $\mathbf{Z}(2)$ подгруппу в $\mathbf{T}$, состоящую из элементов \pm 1 , через $\mathbf{Z}$ - группу целых чисел. Обозначим через $I$ тождественный автоморфизм группы. Положим $Y^{(2)}=\{2 y: y \in Y\}$. Пусть $f(y)$ - функция на группе $Y, h$ - произвольный элемент $Y$. Обозначим через $\Delta_{h}$ оператор конечной разности

$$
\Delta_{h} f(y)=f(y+h)-f(y)
$$

Многочленом на группе $Y$ называется функция $f(y)$ такая, что при некотором $n$ выполнено равенство

$$
\Delta_{h}^{n+1} f(y)=0
$$

для всех $y, h \in Y$. Минимальное $n$, при котором выполнено это равенство, называется степенью многочлена $f(y)$. Если $A$ и $B$ - подмножества в $X$, то положим $A+B=\{x \in X: x=a+b, a \in A, b \in B\}$.

Сверточную полугруппу вероятностных распределений на $X$ обозначим $M^{1}(X)$. Характеристическую функцию распределения $\mu \in$ $M^{1}(X)$ определим формулой

$$
\widehat{\mu}(y)=\int_{X}(x, y) d \mu(x), \quad y \in Y .
$$

Для $\mu \in M^{1}(X)$ определим распределение $\bar{\mu} \in M^{1}(X)$ по формуле $\bar{\mu}(B)=\mu(-B)$ для всех борелевских множеств $B$ в $X$. Заметим, что $\widehat{\bar{\mu}}(y)=\overline{\widehat{\mu}(y)}$. Носитель распределения $\mu \in M^{1}(X)$ обозначим $\sigma(\mu)$. Отметим, что если $H-$ замкнутая подгруппа в $Y$ и $\widehat{\mu}(y)=1$ при $y \in H$, то $\sigma(\mu) \subset A(X, H)$. Будем говорить, что распределение $\mu \in M^{1}(X)$ сосредоточено на борелевском множестве $A \subset X$, если $\mu(B)=0$ для любого борелевского множества $B$ в $X$ такого, что $B \cap A=\varnothing$. Обозначим через $E_{x}$ вырожденное распределение, сосредоточенное в точке $x \in X$.

Распределение $\gamma \in M^{1}(X)$ называется гауссовским ([19], см. также $[20$, гл. IV] $)$, если его характеристическая функция имеет вид

$$
\widehat{\gamma}(y)=(x, y) \exp \{-\varphi(y)\}, \quad y \in Y,
$$

где $x \in X$, а $\varphi(y)$ - непрерывная неотрицательная функция на $Y$, удовлетворяющая уравнению

$$
\varphi(u+v)+\varphi(u-v)=2[\varphi(u)+\varphi(v)], \quad u, v \in Y .
$$

Обозначим через $\Gamma(X)$ множество гауссовских распределений на группе $X$. Отметим, что согласно этому определению вырожденные распределения являются гауссовскими.

Пусть $X=\mathbf{T}$. Тогда $Y \cong \mathbf{Z}$. Чтобы не усложнять обозначения, будем считать, что $Y=\mathbf{Z}$. Пусть $X=\mathbf{R} \times \mathbf{T}$. Элементы группы $X$ будем 
обозначать $x=(t, z), t \in \mathbf{R}, z \in \mathbf{T}$. Если $X=\mathbf{R} \times \mathbf{T}$, то $Y \cong \mathbf{R} \times \mathbf{Z}$. Чтобы не вводить дополнительных обозначений, будем считать, что $Y=\mathbf{R} \times \mathbf{Z}$, а элементы группы $Y$ обозначать $y=(s, n), s \in \mathbf{R}, n \in \mathbf{Z}$. Легко проверить, что каждый автоморфизм $\varepsilon \in \operatorname{Aut}(Y)$ определяется матрицей $\left(\begin{array}{ll}a & c \\ 0 & p\end{array}\right)$, где $a, c \in \mathbf{R}, a \neq 0, p= \pm 1$, и $\varepsilon$ действует на $Y$ следующим образом:

$$
\varepsilon(s, n)=(a s+c n, p n), \quad s \in \mathbf{R}, \quad n \in \mathbf{Z} .
$$

Тогда сопряженный автоморфизм $\delta=\widetilde{\varepsilon} \in \operatorname{Aut}(X)$ имеет вид

$$
\delta(t, z)=\left(a t, e^{i c t} z^{p}\right), \quad t \in \mathbf{R}, \quad z \in \mathbf{T} .
$$

Мы будем отождествлять автоморфизмы $\delta$ и $\varepsilon$ с матрицей $\left(\begin{array}{cc}a & c \\ 0 & p\end{array}\right)$.

Основным результатом настоящей статьи является следующая теорема.

Теорема 1. Пусть $X=\mathbf{R} \times \mathbf{T}, \alpha_{i j} \in \operatorname{Aut}(X), i, j=1,2,3$. Пусть $\xi_{j}, j=1,2,3$, - независимье случайные величинь со значениями в группе $X$ и с распределениями $\mu_{j}$ такими, что их характеристические функиии не обращаются в нуль. Предположим, что линейнье статистики $L_{1}=\alpha_{11} \xi_{1}+\alpha_{12} \xi_{2}+\alpha_{13} \xi_{3}, L_{2}=\alpha_{21} \xi_{1}+\alpha_{22} \xi_{2}+\alpha_{23} \xi_{3}$, $L_{3}=\alpha_{31} \xi_{1}+\alpha_{32} \xi_{2}+\alpha_{33} \xi_{3}$ независимьл. Тогда либо $\mu_{j}-$ вьлрожденнье распределения, либо $\mu_{j}$ - гауссовские распределения, носители которых - классь смежности группь $X$ относительно подаруппь, топологически изоморфной $\mathbf{R}$.

Доказательство теоремы 1 достаточно длинно и опирается на ряд лемм. Поэтому поясним в нескольких словах идею доказательства. Сначала мы доказываем, что в условиях теоремы 1 на произвольной локально компактной абелевой группе $X$ свертка

$$
\nu=\mu_{1} * \bar{\mu}_{1} * \mu_{2} * \bar{\mu}_{2} * \mu_{3} * \bar{\mu}_{3}
$$

является гауссовским распределением. Если группа $X$ не содержит подгруппы, топологически изоморфной $\mathbf{T}$, то на $X$ имеет место групповой аналог теоремы Крамера о разложении гауссовского распределения [21], из которого следует, что и распределения $\mu_{j}$ - гауссовские. Но на группе $X=\mathbf{R} \times \mathbf{T}$ гауссовское распределение может иметь негауссовские делители. Поэтому из того, что распределение $\nu \in M^{1}(\mathbf{R} \times \mathbf{T})$ гауссовское, вообще говоря, не следует, что его делители - также гауссовские. Мы доказываем, что в условиях теоремы 1 гауссовское распределение $\nu$ не произвольно, а сосредоточено на подгруппе, топологически изоморфной $\mathbf{R}$. Отсюда по классической теореме Крамера о разложении гауссовского распределения следует, что распределения $\mu_{j}$ - также гауссовские. 
3. Доказательство лемм. Для доказательства теоремы 1 нам понадобится ряд лемм.

Лемма 1. Пусть $X$ - локально компактная абелева группа, удовлетворяющая второй аксиоме счетности, $\alpha_{i}, \beta_{i} \in \operatorname{Aut}(X), i=1,2$. Пусть $\xi_{j}, j=1,2,3$, - независимье случайнье величинь со значениями в группе $X$ и с распределениями $\mu_{j}$. Для того чтобы линейные статистики $L_{1}=\xi_{1}+\xi_{2}+\xi_{3}, L_{2}=\alpha_{1} \xi_{1}+\alpha_{2} \xi_{2}+\xi_{3}, L_{3}=\beta_{1} \xi_{1}+\beta_{2} \xi_{2}+\xi_{3}$ бьли независимьми, необходимо и достаточно, чтобы характеристические функиии $\widehat{\mu}_{j}(y)$ удовлетворяли уравнению

$$
\begin{aligned}
& \widehat{\mu}_{1}\left(u+\widetilde{\alpha}_{1} v+\widetilde{\beta}_{1} w\right) \widehat{\mu}_{2}\left(u+\widetilde{\alpha}_{2} v+\widetilde{\beta}_{2} w\right) \widehat{\mu}_{3}(u+v+w) \\
& \quad=\widehat{\mu}_{1}(u) \widehat{\mu}_{2}(u) \widehat{\mu}_{3}(u) \widehat{\mu}_{1}\left(\widetilde{\alpha}_{1} v\right) \widehat{\mu}_{2}\left(\widetilde{\alpha}_{2} v\right) \widehat{\mu}_{3}(v) \widehat{\mu}_{1}\left(\widetilde{\beta}_{1} w\right) \widehat{\mu}_{2}\left(\widetilde{\beta}_{2} w\right) \widehat{\mu}_{3}(w),
\end{aligned}
$$

где $u, v, w \in Y$.

Д о к а з а т е л ь с т в о. Отметим, что если $\xi$ - случайная величина со значениями в группе $X$ и с распределением $\mu$, то $\widehat{\mu}(y)=\mathbf{E}[(\xi, y)]$. Очевидно, что линейные статистики $L_{1}, L_{2}$ и $L_{3}$ независимы тогда и только тогда, когда для любых $u, v, w \in Y$ выполнено равенство

$$
\begin{aligned}
& \mathbf{E}\left[\left(\xi_{1}+\xi_{2}+\xi_{3}, u\right)\left(\alpha_{1} \xi_{1}+\alpha_{2} \xi_{2}+\xi_{3}, v\right)\left(\beta_{1} \xi_{1}+\beta_{2} \xi_{2}+\xi_{3}, w\right)\right] \\
& =\mathbf{E}\left[\left(\xi_{1}+\xi_{2}+\xi_{3}, u\right)\right] \mathbf{E}\left[\left(\alpha_{1} \xi_{1}+\alpha_{2} \xi_{2}+\xi_{3}, v\right)\right] \\
& \quad \times \mathbf{E}\left[\left(\beta_{1} \xi_{1}+\beta_{2} \xi_{2}+\xi_{3}, w\right)\right] .
\end{aligned}
$$

Учитывая то, что случайные величины $\xi_{j}$ независимы, преобразуем левую часть равенства (3) следующим образом:

$$
\begin{aligned}
\mathbf{E} & {\left[\left(\xi_{1}+\xi_{2}+\xi_{3}, u\right)\left(\alpha_{1} \xi_{1}+\alpha_{2} \xi_{2}+\xi_{3}, v\right)\left(\beta_{1} \xi_{1}+\beta_{2} \xi_{2}+\xi_{3}, w\right)\right] } \\
& =\mathbf{E}\left[\left(\xi_{1}, u+\widetilde{\alpha}_{1} v+\widetilde{\beta}_{1} w\right)\left(\xi_{2}, u+\widetilde{\alpha}_{2} v+\widetilde{\beta}_{2} w\right)\left(\xi_{3}, u+v+w\right)\right] \\
& =\mathbf{E}\left[\left(\xi_{1}, u+\widetilde{\alpha}_{1} v+\widetilde{\beta}_{1} w\right)\right] \mathbf{E}\left[\left(\xi_{2}, u+\widetilde{\alpha}_{2} v+\widetilde{\beta}_{2} w\right)\right] \mathbf{E}\left[\left(\xi_{3}, u+v+w\right)\right] \\
& =\widehat{\mu}_{1}\left(u+\widetilde{\alpha}_{1} v+\widetilde{\beta}_{1} w\right) \widehat{\mu}_{2}\left(u+\widetilde{\alpha}_{2} v+\widetilde{\beta}_{2} w\right) \widehat{\mu}_{3}(u+v+w) .
\end{aligned}
$$

Аналогично преобразуем правую часть равенства (3):

$$
\begin{aligned}
\mathbf{E}[ & \left.\left(\xi_{1}+\xi_{2}+\xi_{3}, u\right)\right] \mathbf{E}\left[\left(\alpha_{1} \xi_{1}+\alpha_{2} \xi_{2}+\xi_{3}, v\right)\right] \mathbf{E}\left[\left(\beta_{1} \xi_{1}+\beta_{2} \xi_{2}+\xi_{3}, w\right)\right] \\
= & \mathbf{E}\left[\left(\xi_{1}, u\right)\left(\xi_{2}, u\right)\left(\xi_{3}, u\right)\right] \mathbf{E}\left[\left(\xi_{1}, \widetilde{\alpha}_{1} v\right)\left(\xi_{2}, \widetilde{\alpha}_{2} v\right)\left(\xi_{3}, v\right)\right] \\
& \times \mathbf{E}\left[\left(\xi_{1}, \widetilde{\beta}_{1} w\right)\left(\xi_{2}, \widetilde{\beta}_{2} w\right)\left(\xi_{3}, w\right)\right] \\
= & \mathbf{E}\left[\left(\xi_{1}, u\right)\right] \mathbf{E}\left[\left(\xi_{2}, u\right)\right] \mathbf{E}\left[\left(\xi_{3}, u\right)\right] \mathbf{E}\left[( \xi _ { 1 } , \widetilde { \alpha } _ { 1 } v ) \mathbf { E } \left[\left(\xi_{2}, \widetilde{\alpha}_{2} v\right)\right.\right. \\
& \times \mathbf{E}\left[\left(\xi_{3}, v\right)\right] \mathbf{E}\left[\left(\xi_{1}, \widetilde{\beta}_{1} w\right)\right] \mathbf{E}\left[\left(\xi_{2}, \widetilde{\beta}_{2} w\right)\right] \mathbf{E}\left[\left(\xi_{3}, w\right)\right] \\
= & \widehat{\mu}_{1}(u) \widehat{\mu}_{2}(u) \widehat{\mu}_{3}(u) \widehat{\mu}_{1}\left(\widetilde{\alpha}_{1} v\right) \widehat{\mu}_{2}\left(\widetilde{\alpha}_{2} v\right) \widehat{\mu}_{3}(v) \widehat{\mu}_{1}\left(\widetilde{\beta}_{1} w\right) \widehat{\mu}_{2}\left(\widetilde{\beta}_{2} w\right) \widehat{\mu}_{3}(w) .
\end{aligned}
$$

Лемма 1 доказана. 
Лемма 2. Пусть $\xi_{j}$ - независимье случайнье величинь со значениями в группе $\mathbf{R}$ и с распределениями $\mu_{j}$. Пусть $a_{j}, b_{j}, j=1,2,-$ ненулевье константы. Предположим, что линейные статистики $L_{1}=\xi_{1}+\xi_{2}+\xi_{3}, L_{2}=a_{1} \xi_{1}+a_{2} \xi_{2}+\xi_{3}, L_{3}=b_{1} \xi_{1}+b_{2} \xi_{2}+\xi_{3}$ независимы. Тогда либо все $\mu_{j}$ - вырожденнье распределения, либо все $\mu_{j}-$ невырожденнье гауссовские распределения, и справедливь следующие утверждения:

1) $a_{1} b_{2}-a_{1} a_{2} b_{2}-a_{1} b_{1} b_{2}-a_{2} b_{1}+a_{1} a_{2} b_{1}+a_{2} b_{1} b_{2}=0$

2) возможнье комбинаиии знаков $a_{i}, b_{i}$ описьваются в табличе

\begin{tabular}{|c|c|c|c|}
\hline$a_{1}$ & $a_{2}$ & $b_{1}$ & $b_{2}$ \\
\hline+ & - & - & + \\
+ & - & - & - \\
- & + & + & - \\
- & + & - & - \\
- & - & + & - \\
- & - & - & + \\
\hline
\end{tabular}

3) $a_{1} \neq a_{2}, b_{1} \neq b_{2}$;

4) $a_{2} b_{1}-a_{1} b_{2} \neq 0$

5) $\left|\begin{array}{ll}a_{1}-1 & a_{2}-1 \\ b_{1}-1 & b_{2}-1\end{array}\right| \neq 0$.

Д о к а з а т е л ь с т в о. По лемме 1 характеристические функции $\widehat{\mu}_{j}(y)$ удовлетворяют уравнению (2), которое принимает вид

$$
\begin{aligned}
& \widehat{\mu}_{1}\left(u+a_{1} v+b_{1} w\right) \widehat{\mu}_{2}\left(u+a_{2} v+b_{2} w\right) \widehat{\mu}_{3}(u+v+w) \\
& \quad=\widehat{\mu}_{1}(u) \widehat{\mu}_{2}(u) \widehat{\mu}_{3}(u) \widehat{\mu}_{1}\left(a_{1} v\right) \widehat{\mu}_{2}\left(a_{2} v\right) \widehat{\mu}_{3}(v) \widehat{\mu}_{1}\left(b_{1} w\right) \widehat{\mu}_{2}\left(b_{2} w\right) \widehat{\mu}_{3}(w),
\end{aligned}
$$

где $u, v, w \in \mathbf{R}$. Из независимости линейных статистик $L_{1}$ и $L_{2}$ по теореме Скитовича-Дармуа вытекает, что все $\mu_{j}$ - гауссовские распределения, т.е. характеристические функции $\widehat{\mu}_{j}(s)$ представимы в виде

$$
\widehat{\mu}_{j}(s)=\exp \left\{-\sigma_{j} s^{2}+i \tau_{j} s\right\}, \quad s \in \mathbf{R},
$$

где $\sigma_{j} \geqslant 0, \tau_{j} \in \mathbf{R}$. Подставляя (5) в (4), получаем, что справедливы следующие равенства:

$$
\begin{aligned}
\sigma_{1} a_{1}+\sigma_{2} a_{2}+\sigma_{3} & =0, \\
\sigma_{1} b_{1}+\sigma_{2} b_{2}+\sigma_{3} & =0, \\
\sigma_{1} a_{1} b_{1}+\sigma_{2} a_{2} b_{2}+\sigma_{3} & =0 .
\end{aligned}
$$

Из (6)-(8) легко вытекает, что либо все $\mu_{j}$ - вырожденные распределения, либо все $\mu_{j}$ - невырожденные распределения.

Предположим, что распределения $\mu_{j}$ невырожденные. Тогда в (6)(8) все $\sigma_{j}$ положительны. Докажем утверждения 1)-5). 
1) Так как в (6)-(8) все $\sigma_{j}$ отличны от нуля, то

$$
\left|\begin{array}{ccc}
a_{1} & a_{2} & 1 \\
b_{1} & b_{2} & 1 \\
a_{1} b_{1} & a_{2} b_{2} & 1
\end{array}\right|=0
$$

т.е. выполнено 1).

2) Так как все $\sigma_{j}$ положительны, то 2) непосредственно вытекает из (6) $-(8)$.

3) Предположим, что $a_{1}=a_{2}$. Тогда из 2) следует, что $a_{i}<0$, а из $(7)$ и (8) вытекает, что $\left(1-a_{1}\right) \sigma_{3}=0$. Так как $a_{1}<0$, то отсюда следует, что $\sigma_{3}=0$. Тогда из (6) получаем, что $\sigma_{1}=\sigma_{2}=0$. Полученное противоречие доказывает 3) в случае, когда $a_{1}=a_{2}$. Аналогично рассуждаем в случае, когда $b_{1}=b_{2}$.

4) Предположим, что $a_{2} b_{1}-a_{1} b_{2}=0$. Тогда

$$
b_{2}=\frac{a_{2} b_{1}}{a_{1}} \text {. }
$$

Учитывая (9), перепишем (7) в виде

$$
\frac{b_{1}}{a_{1}}\left(\sigma_{1} a_{1}+\sigma_{2} a_{2}\right)+\sigma_{3}=0 .
$$

Отсюда и из (6) вытекает, что

$$
\left(1-\frac{b_{1}}{a_{1}}\right) \sigma_{3}=0 .
$$

С учетом 2) из (9) следует, что либо $a_{1}>0, a_{2}<0, b_{1}<0, b_{2}>0$, либо $a_{1}<0, a_{2}>0, b_{1}>0, b_{2}<0$. Поскольку в обоих случаях $a_{1} b_{1}<0$, то из (10) следует, что $\sigma_{3}=0$. Тогда, учитывая что $a_{i}, b_{i}<0$, из (8) получаем, что $\sigma_{1}=\sigma_{2}=0$. Полученное противоречие доказывает 4 ).

5) Предположим, что $\left|\begin{array}{ll}a_{1}-1 & a_{2}-1 \\ b_{1}-1 & b_{2}-1\end{array}\right|=0$, т.е.

$$
\left(a_{1}-1\right)\left(b_{2}-1\right)=\left(a_{2}-1\right)\left(b_{1}-1\right) \text {. }
$$

Учитывая (11), перепишем левую часть равенства 1) следующим обра30M:

$$
\begin{aligned}
a_{1} b_{2} & -a_{1} a_{2} b_{2}-a_{1} b_{1} b_{2}+a_{1} a_{2} b_{1} b_{2}-a_{2} b_{1}+a_{1} a_{2} b_{1}+a_{2} b_{1} b_{2}-a_{1} a_{2} b_{1} b_{2} \\
& =a_{1} b_{2}\left(1-a_{2}-b_{1}+a_{2} b_{1}\right)-a_{2} b_{1}\left(1-a_{1}-b_{2}+a_{1} b_{2}\right) \\
& =a_{1} b_{2}\left(a_{2}-1\right)\left(b_{1}-1\right)-a_{2} b_{1}\left(a_{1}-1\right)\left(b_{2}-1\right) \\
& =\left(a_{2}-1\right)\left(b_{1}-1\right)\left(a_{1} b_{2}-a_{2} b_{1}\right)=0 .
\end{aligned}
$$

Так как согласно 4) $a_{2} b_{1}-a_{1} b_{2} \neq 0$, то либо $a_{2}=1$, либо $b_{1}=1$. Как следует из 2), в этих случаях $a_{1}<0, b_{2}<0$. Мы получили противоречие с (11). Лемма доказана. 
Лемма 3 (см. $[22, \S 2])$. Пусть $X$ - топологическая абелева групnа, $G$ - борелевская подгруппа в $X, \mu \in M^{1}(G), \mu=\mu_{1} * \mu_{2}$. Тогда распределения $\mu_{j}$ можно заменить их сдвигами $\mu_{j}^{\prime}$ таким образом, что $\mu=\mu_{1}^{\prime} * \mu_{2}^{\prime}, u \mu_{j}^{\prime} \in M^{1}(G)$.

Лемма 4 (см. [18]). Пусть $X=\mathbf{T}, a \xi_{1} u \xi_{2}$ - независимьге случайные величинь со значениями в группе $X=\mathbf{T}$ и с распределениями $\mu_{j}$, имеюшими не обращающиеся в нуль характеристические функции. Предположим, что сумма $\xi_{1}+\xi_{2}$ и разность $\xi_{1}-\xi_{2}$ независимь. Тогда $\mu_{j}=E_{x_{j}} * \gamma * \pi_{j}$, где $x_{j} \in \mathbf{T}, \gamma \in \Gamma(\mathbf{T}), \pi_{j}-$ зарядын на $\mathbf{Z}(2)$ такие, что $\pi_{1} * \pi_{2}=E_{1}$. Другими словами, характеристические функиии $\widehat{\mu}_{j}(n)$ представимь в виде

$$
\begin{array}{ll}
\widehat{\mu}_{1}(n)=\exp \left\{-\sigma n^{2}+i \theta_{1} n+\kappa\left(1-(-1)^{n}\right)\right\}, & n \in \mathbf{Z}, \\
\widehat{\mu}_{2}(n)=\exp \left\{-\sigma n^{2}+i \theta_{2} n-\kappa\left(1-(-1)^{n}\right)\right\}, & n \in \mathbf{Z},
\end{array}
$$

где $\sigma \geqslant 0, \kappa \in \mathbf{R}, 0 \leqslant \theta_{j}<2 \pi, j=1,2$.

Лемма 5. Пусть $X=\mathbf{T}, a \xi_{j}, j=1,2,3$, - независимье случайнье величинь со значениями в группе $X$ и с распределениями $\mu_{j}$, имеющими необращающиеся в нуль характеристические функиии. Пусть $\alpha_{i j} \in$ $\operatorname{Aut}(X), i, j=1,2,3$. Тогда из независимости линейньх статистик $L_{1}=\alpha_{11} \xi_{1}+\alpha_{12} \xi_{2}+\alpha_{13} \xi_{3}, L_{2}=\alpha_{21} \xi_{1}+\alpha_{22} \xi_{2}+\alpha_{23} \xi_{3}, L_{3}=\alpha_{31} \xi_{1}+\alpha_{32} \xi_{2}+$ $\alpha_{33} \xi_{3}$ вылекает, что все $\mu_{j}$ - вырожденнье распределения.

Д о к а з а т е л ь с в о. Очевидно, что Aut $(X)=\{I,-I\}$. Заметим, что если для каких-нибудь двух линейных статистик, например $L_{1}$ и $L_{2}$, выполнено $L_{1}= \pm L_{2}$, то все $\mu_{j}$ - вырожденные распределения. Поэтому мы можем предполагать, что $L_{i} \neq \pm L_{j}$ для всех $i, j=1,2,3$, $i \neq j$. Отсюда легко следует, что без ограничения общности можно считать, что $L_{1}=\xi_{1}+\xi_{2}+\xi_{3}, L_{2}=\xi_{1}-\xi_{2}+\xi_{3}, L_{3}=-\xi_{1}+\xi_{2}+\xi_{3}$.

Обозначим $\eta_{1}=\xi_{1}+\xi_{3}, \eta_{2}=\xi_{2}$. Тогда $\eta_{1}, \eta_{2}$ - независимые случайные величины. Из независимости $L_{1}$ и $L_{2}$ следует, что сумма $\eta_{1}+\eta_{2}$ и разность $\eta_{1}-\eta_{2}$ также независимы. Из леммы 4 , примененной к независимым случайным величинам $\eta_{1}$ и $\eta_{2}$, вытекает, что существует $\sigma_{2} \geqslant 0$ такое, что

$$
\left|\widehat{\mu}_{1}(2 n)\right|\left|\widehat{\mu}_{3}(2 n)\right|=\left|\widehat{\mu}_{2}(2 n)\right|=e^{-4 \sigma_{2} n^{2}}, \quad n \in \mathbf{Z} .
$$

Аналогично, из независимости линейных статистик $L_{1}, L_{3}$ и линейных статистик $-L_{2}, L_{3}$ получаем, что существуют $\sigma_{1} \geqslant 0, \sigma_{3} \geqslant 0$ такие, что

$$
\begin{aligned}
& \left|\widehat{\mu}_{2}(2 n)\right|\left|\widehat{\mu}_{3}(2 n)\right|=\left|\widehat{\mu}_{1}(2 n)\right|=e^{-4 \sigma_{1} n^{2}}, \quad n \in \mathbf{Z}, \\
& \left|\widehat{\mu}_{1}(2 n)\right|\left|\widehat{\mu}_{2}(2 n)\right|=\left|\widehat{\mu}_{3}(2 n)\right|=e^{-4 \sigma_{3} n^{2}}, \quad n \in \mathbf{Z} \text {. }
\end{aligned}
$$

Из (14)-(16) вытекает, что

$$
\sigma_{1}+\sigma_{3}=\sigma_{2}, \quad \sigma_{2}+\sigma_{3}=\sigma_{1}, \quad \sigma_{1}+\sigma_{2}=\sigma_{3} .
$$


Отсюда следует, что

$$
\sigma_{1}=\sigma_{2}=\sigma_{3}=0
$$

Поскольку $\widehat{\mu}_{j}(n)$ - характеристические функции распределений, то из (17) вытекает, что в $(12) \kappa=0$. Значит, $\mu_{j}$ - вырожденные распределения. Лемма доказана.

Пусть $X$ - локально компактная абелева группа, $\alpha_{i}, \beta_{i} \in \operatorname{Aut}(X)$, $i=1,2$. Введем следующие обозначения, которые сохраним на протяжении всей статьи:

$$
\begin{gathered}
L=\left(\widetilde{\alpha}_{1}-I\right) Y+\left(\widetilde{\beta}_{1}-I\right) Y, \quad M=\left(\widetilde{\alpha}_{2}-I\right) Y+\left(\widetilde{\beta}_{2}-I\right) Y \\
N=\left(\widetilde{\alpha}_{2}-\widetilde{\alpha}_{1}\right) Y+\left(\widetilde{\beta}_{2}-\widetilde{\beta}_{1}\right) Y .
\end{gathered}
$$

Лемма 6. Пусть $X$ - локально компактная абелева группа, удовлетворяющая второй аксиоме счетности, $\alpha_{i}, \beta_{i} \in \operatorname{Aut}(X), i=1,2$. Пусть $\xi_{j}, j=1,2,3$, - независимьле случайнье величинь со значениями в группе $X$ и с распределениями $\mu_{j}$, имеющими не обращающиеся 8 нуль характеристические функиии. Предположим, что линейнье статистики $L_{1}=\xi_{1}+\xi_{2}+\xi_{3}, L_{2}=\alpha_{1} \xi_{1}+\alpha_{2} \xi_{2}+\xi_{3}, L_{3}=\beta_{1} \xi_{1}+\beta_{2} \xi_{2}+\xi_{3}$ независимы. Положим $\nu_{j}=\mu_{j} * \bar{\mu}_{j}$. Тогда $\nu=\nu_{1} * \nu_{2} * \nu_{3} \in \Gamma(X), a$ функиии $\psi_{j}(y)=-\ln \widehat{\nu}_{j}(y)$ удовлетворяют уравнениям

$$
\begin{array}{llll}
\Delta_{h} \Delta_{k} \Delta_{l} \psi_{1}(y)=0, & h, y \in Y, & k \in N, & l \in L, \\
\Delta_{h} \Delta_{k} \Delta_{l} \psi_{2}(y)=0, & h, y \in Y, & k \in N, & l \in M, \\
\Delta_{h} \Delta_{k} \Delta_{l} \psi_{3}(y)=0, & h, y \in Y, & k \in L, & l \in M .
\end{array}
$$

Д о к а з а т е л ь с т в о. По лемме 1 характеристические функции $\widehat{\mu}_{j}(y)$ удовлетворяют уравнению (2). Заметим, что $\widehat{\nu}_{j}(y)=\left|\widehat{\mu}_{j}(y)\right|^{2}>$ $0, y \in Y$, и характеристические функции $\widehat{\nu}_{j}(y)$ также удовлетворяют уравнению (2). Из уравнения (2) получаем

$$
\begin{gathered}
\psi_{1}\left(u+\widetilde{\alpha}_{1} v+\widetilde{\beta}_{1} w\right)+\psi_{2}\left(u+\widetilde{\alpha}_{2} v+\widetilde{\beta}_{2} w\right)+\psi_{3}(u+v+w) \\
=A(u)+B(v)+C(w), \quad u, v, w \in Y,
\end{gathered}
$$

где

$$
\begin{aligned}
& A(y)=\psi_{1}(y)+\psi_{2}(y)+\psi_{3}(y), \\
& B(y)=\psi_{1}\left(\widetilde{\alpha}_{1} y\right)+\psi_{2}\left(\widetilde{\alpha}_{2} y\right)+\psi_{3}(y), \\
& C(y)=\psi_{1}\left(\widetilde{\beta}_{1} y\right)+\psi_{2}\left(\widetilde{\beta}_{2} y\right)+\psi_{3}(y) .
\end{aligned}
$$

Для доказательства леммы воспользуемся методом конечных разностей. Пусть $k_{1}$ и $m_{1}$ - произвольные элементы группы $Y$. Положим $h_{1}=$ $-k_{1}-m_{1}$. Тогда $h_{1}+k_{1}+m_{1}=0$. Придадим в (21) переменным $u, v$ 
и $w$ приращения $h_{1}, k_{1}$ и $m_{1}$ соответственно. Вычитая из полученного уравнения уравнение (21), находим

$$
\begin{aligned}
\Delta_{l_{11}} \psi_{1}(u & \left.+\widetilde{\alpha}_{1} v+\widetilde{\beta}_{1} w\right)+\Delta_{l_{12}} \psi_{2}\left(u+\widetilde{\alpha}_{2} v+\widetilde{\beta}_{2} w\right) \\
= & \Delta_{h_{1}} A(u)+\Delta_{k_{1}} B(v)+\Delta_{m_{1}} C(w), \quad u, v, w \in Y
\end{aligned}
$$

где $l_{11}=\left(\widetilde{\alpha}_{1}-I\right) k_{1}+\left(\widetilde{\beta}_{1}-I\right) m_{1}, l_{12}=\left(\widetilde{\alpha}_{2}-I\right) k_{1}+\left(\widetilde{\beta}_{2}-I\right) m_{1}$. Пусть $k_{2}$ и $m_{2}$ - произвольные элементы группы $Y$. Положим $h_{2}=-\widetilde{\alpha}_{2} k_{2}-$ $\widetilde{\beta}_{2} m_{2}$. Тогда $h_{2}+\widetilde{\alpha}_{2} k_{2}+\widetilde{\beta}_{2} m_{2}=0$. Придадим в $(22)$ переменным $u, v$ и $w$ приращения $h_{2}, k_{2}$ и $m_{2}$ соответственно. Вычитая из полученного уравнения уравнение $(22)$, получаем

$$
\begin{aligned}
& \Delta_{l_{21}} \Delta_{l_{11}} \psi_{1}\left(u+\widetilde{\alpha}_{1} v+\widetilde{\beta}_{1} w\right)=\Delta_{h_{2}} \Delta_{h_{1}} A(u) \\
& \quad+\Delta_{k_{2}} \Delta_{k_{1}} B(v)+\Delta_{m_{2}} \Delta_{m_{1}} C(w), \quad u, v, w \in Y,
\end{aligned}
$$

где $l_{21}=\left(\widetilde{\alpha}_{2}-\widetilde{\alpha}_{1}\right) k_{2}+\left(\widetilde{\beta}_{2}-\widetilde{\beta}_{1}\right) m_{2}$. Пусть $k_{3}$ и $m_{3}-$ произвольные элементы группы $Y$. Положим $h_{3}=-\widetilde{\alpha}_{1} k_{3}-\widetilde{\beta}_{1} m_{3}$. Тогда $h_{3}+\widetilde{\alpha}_{1} k_{3}+$ $\widetilde{\beta}_{1} m_{3}=0$. Придадим в (23) переменным $u, v$ и $w$ приращения $h_{3}, k_{3}$ и $m_{3}$ соответственно. Вычитая из полученного уравнения уравнение $(23)$, находим

$\Delta_{h_{3}} \Delta_{h_{2}} \Delta_{h_{1}} A(u)+\Delta_{k_{3}} \Delta_{k_{2}} \Delta_{k_{1}} B(v)+\Delta_{m_{3}} \Delta_{m_{2}} \Delta_{m_{1}} C(w)=0, \quad u, v, w \in Y$.

Пусть $h_{4}$ - произвольный элемент группы $Y$. Придадим в (24) переменной $u$ приращение $h_{4}$. Вычитая из полученного уравнения уравнение (24), получаем

$$
\Delta_{h_{4}} \Delta_{h_{3}} \Delta_{h_{2}} \Delta_{h_{1}} A(u)=0, \quad h_{j}, u \in Y .
$$

Полагая в $(25) h_{1}=h_{2}=h_{3}=h_{4}=h$, находим

$$
\Delta_{h}^{4} A(u)=0, \quad h, u \in Y .
$$

Таким образом, $A(y)$ - многочлен степени $\leqslant 3$. Тогда существуют симметричные $k$-аддитивные функции $g_{k}\left(y_{1}, y_{2}, \ldots, y_{k}\right), k=1,2,3$, такие, чTO

$$
A(y)=g_{3}^{*}(y)+g_{2}^{*}(y)+g_{1}^{*}(y)+g_{0}, \quad y \in Y
$$

где $g_{k}^{*}(y)=g_{k}(y, \ldots, y), g_{0}=$ const [23]. Очевидно, что $g_{k}^{*}(-y)=$ $(-1)^{k} g_{k}^{*}(y)$. Так как $A(-y)=A(y)$ и $A(0)=0$, то $A(y)=g_{2}^{*}(y)$. Нетрудно проверить, что функция $g_{2}^{*}(y)$ удовлетворяет уравнению (1). Следовательно, $\nu \in \Gamma(X)$.

Так как $A(y)=g_{2}^{*}(y)=g_{2}(y, y)$, то легко видеть, что функция $A(y)$ удовлетворяет уравнению

$$
\Delta_{h_{3}} \Delta_{h_{2}} \Delta_{h_{1}} A(u)=0, \quad h_{j}, u \in Y .
$$


Аналогично получаем, что функции $B(v)$ и $C(w)$ также удовлетворяют уравнению (27).

Пусть $h-$ произвольный элемент группы $Y$. Придадим в $(23)$ переменной $u$ приращение $h$. Вычитая из полученного уравнения уравнение (23) и учитывая (27), находим

$$
\Delta_{h} \Delta_{l_{21}} \Delta_{l_{11}} \psi_{1}\left(u+\widetilde{\alpha}_{1} v+\widetilde{\beta}_{1} w\right)=0 .
$$

Полагая здесь $v=w=0$ и учитывая выражения для $l_{11}$ и $l_{21}$, получаем, что функция $\psi_{1}(y)$ удовлетворяет уравнению (18). Рассуждая аналогично, убеждаемся, что функция $\psi_{2}(y)$ удовлетворяет уравнению (19), а функция $\psi_{3}(y)$ удовлетворяет уравнению (20). Лемма доказана.

Лемма 7. Пусть $X=\mathbf{R} \times \mathbf{T} u \alpha_{i}=\left(\begin{array}{cc}a_{i} & c_{i} \\ 0 & p_{i}\end{array}\right), \beta_{i}=\left(\begin{array}{cc}b_{i} & d_{i} \\ 0 & q_{i}\end{array}\right)$, $i=1,2$, - топологические автоморфизмь группь $X$. Пусть $\xi_{j}, j=$ $1,2,3$, - независимые случайные величины со значениями в группе $X$ и с распределениями $\mu_{j}$. Предположим, что линейнье статистики $L_{1}=\xi_{1}+\xi_{2}+\xi_{3}, L_{2}=\alpha_{1} \xi_{1}+\alpha_{2} \xi_{2}+\xi_{3}, L_{3}=\beta_{1} \xi_{1}+\beta_{2} \xi_{2}+\xi_{3}$ независимьл. Тогда либо все $\mu_{j}$ - вырожденные распределения, либо все $\mu_{j}-$ невырожденные распределения, и для подгрупп $L, M$ и $N$ имеются такие возможности:

$$
\begin{array}{llll}
\text { 1. } & L=\mathbf{R}, & M=\mathbf{R}, & N=\mathbf{R} ; \\
\text { 2. } & L=\mathbf{R}, & M=Y^{(2)}, & N=Y^{(2)} ; \\
\text { 3. } & L=Y^{(2)}, & M=\mathbf{R}, & N=Y^{(2)} ; \\
\text { 4. } & L=Y^{(2)}, & M=Y^{(2)}, & N=\mathbf{R} ; \\
\text { 5. } & L=Y^{(2)}, & M=Y^{(2)}, & N=Y^{(2)}
\end{array}
$$

Д о к а $з$ а т е л ь с т в о. По лемме 1 характеристические функции $\widehat{\mu}_{j}(y)$ удовлетворяют уравнению (2). Положим в $(2) u=\left(s_{1}, 0\right), v=$ $\left(s_{2}, 0\right), w=\left(s_{3}, 0\right)$. Получим

$$
\begin{aligned}
& \widehat{\mu}_{1}\left(s_{1}+a_{1} s_{2}+b_{1} s_{3}, 0\right) \widehat{\mu}_{2}\left(s_{1}+a_{2} s_{2}+b_{2} s_{3}, 0\right) \widehat{\mu}_{3}\left(s_{1}+s_{2}+s_{3}, 0\right) \\
& =\widehat{\mu}_{1}\left(s_{1}, 0\right) \widehat{\mu}_{2}\left(s_{1}, 0\right) \widehat{\mu}_{3}\left(s_{1}, 0\right) \widehat{\mu}_{1}\left(a_{1} s_{2}, 0\right) \widehat{\mu}_{2}\left(a_{2} s_{2}, 0\right) \widehat{\mu}_{3}\left(s_{2}, 0\right) \\
& \quad \times \widehat{\mu}_{1}\left(b_{1} s_{3}, 0\right) \widehat{\mu}_{2}\left(b_{2} s_{3}, 0\right) \widehat{\mu}_{3}\left(s_{3}, 0\right), \quad s_{1}, s_{2}, s_{3} \in \mathbf{R} .
\end{aligned}
$$

Положим $\nu_{j}=\mu_{j} * \bar{\mu}_{j}$. Тогда $\widehat{\nu}_{j}(y)=\left|\widehat{\mu}_{j}(y)\right|^{2}>0$ при $y \in Y$. Очевидно, что функции $\widehat{\nu}_{j}(y)$ также удовлетворяют уравнению (2), а значит, и уравнению (29). Из (29) по лемме 1 , примененной к группе $\mathbf{R}$, и лемме 2 следует, что если хотя бы одна из функций $\widehat{\nu}_{j}(s, 0)-$ характеристическая функция невырожденного распределения, то все $\widehat{\nu}_{j}(s, 0)-$ характеристические функции невырожденных гауссовских распределений. Другими словами, либо $\widehat{\nu}_{j}(s, 0)=1$ при $s \in \mathbf{R}, j=1,2,3$, либо $\widehat{\nu}_{j}(s, 0)=e^{-\sigma_{j} s^{2}}$, где $\sigma_{j}>0, j=1,2,3$. 
Предположим, что $\widehat{\nu}_{j}(s, 0)=1$ при $s \in \mathbf{R}, j=1,2,3$. Тогда имеют место включения $\sigma\left(\nu_{j}\right) \subset A(X, \mathbf{R})=\mathbf{T}, j=1,2,3$. Следовательно, по лемме 3 распределения $\mu_{j}$ можно так заменить их сдвигами $\mu_{j}^{\prime}$, что $\sigma\left(\mu_{j}^{\prime}\right) \subset \mathbf{T}, j=1,2,3$. Так как подгруппа $\mathbf{T}$ инвариантна относительно любого топологического автоморфизма группы $X$, то в этом случае по лемме 5 все распределения $\mu_{j}$ вырожденные.

Предположим теперь, что $\widehat{\nu}_{j}(s, 0)=e^{-\sigma_{j} s^{2}}$, где $\sigma_{j}>0, j=1,2,3$. Тогда из утверждения 2) леммы 2 вытекает, что выполнены условия:

(i) либо $a_{1} \neq 1$, либо $b_{1} \neq 1 ; \quad$ (ii) либо $a_{2} \neq 1$, либо $b_{2} \neq 1$.

Заметим теперь, что из (i) следует, что для $L$ имеются только две возможности: либо $L=\mathbf{R}$, либо $L=Y^{(2)}$. Аналогично, из (ii) вытекает, что для $M$ имеются только две возможности: либо $M=\mathbf{R}$, либо $M=$ $Y^{(2)}$. Очевидно, что каждая из этих возможностей реализуется.

Пусть $L=\mathbf{R}$ и $M=\mathbf{R}$. Тогда $p_{i}=q_{i}=1, i=1,2$. Следовательно, $p_{2}-p_{1}=0$, и $q_{2}-q_{1}=0$. Учитывая утверждение 3 ) леммы 2 , отсюда получаем, что для $N$ имеется единственная возможность $N=\mathbf{R}$.

Пусть $L=\mathbf{R}$ и $M=Y^{(2)}$. Тогда $p_{1}=q_{1}=1$ и либо $p_{2}=-1$, либо $q_{2}=-1$. Следовательно, либо $p_{2}-p_{1}=-2$, либо $q_{2}-q_{1}=-2$. Учитывая утверждение 3 ) леммы 2 , отсюда получаем, что для $N$ в таком случае имеется единственная возможность $N=Y^{(2)}$.

Пусть $L=Y^{(2)}$ и $M=\mathbf{R}$. Тогда либо $p_{1}=-1$, либо $q_{1}=-1$, и $p_{2}=q_{2}=1$. Следовательно, либо $p_{2}-p_{1}=2$, либо $q_{2}-q_{1}=2$. Учитывая утверждение 3 ) леммы 2 , отсюда получаем, что для $N$ также имеется единственная возможность $N=Y^{(2)}$.

Пусть $L=Y^{(2)}$ и $M=Y^{(2)}$. Тогда либо $p_{1}=-1$, либо $q_{1}=-1$, и либо $p_{2}=-1$, либо $q_{2}=-1$. Если при этом $p_{1}=p_{2}$ и $q_{1}=q_{2}$, то в силу утверждения 3 ) леммы $2 N=\mathbf{R}$. Если же либо $p_{1} \neq p_{2}$, либо $q_{1} \neq q_{2}$, то в силу утверждения 3 ) леммы $2, N=Y^{(2)}$. Лемма доказана.

Лемма 8. Пусть $Y=\mathbf{R} \times \mathbf{Z}$ и непрерьвная функиия $f(y), y=$ $(s, n) \in Y$, удовлетворяет уравнению

$$
\Delta_{k}^{2} \Delta_{h} f(y)=0, \quad k \in \mathbf{R}, \quad h, y \in Y .
$$

Предположим также, что $f(-y)=f(y), y \in Y$. Тогда

$$
f(s, n)=\sigma s^{2}+\kappa(n) s+\lambda(n), \quad s \in \mathbf{R}, \quad n \in \mathbf{Z},
$$

где $\kappa(-n)=-\kappa(n)$ u $\lambda(-n)=\lambda(n), n \in \mathbf{Z}$.

Д о к а з а т е л ь с т в о. Обозначим $f_{n}(s)=f(s, n), s \in \mathbf{R}, n \in \mathbf{Z}$. Полагая в (30) $y=(s, n), k=h=(t, 0)$, получаем, что функция $f_{n}(s)$ при каждом фиксированном $n$ удовлетворяет уравнению

$$
\Delta_{t}^{3} f_{n}(s)=0, \quad t, s \in \mathbf{R} .
$$


Из (32) вытекает, что функция $f_{n}(s)$ имеет вид

$$
f_{n}(s)=\sigma(n) s^{2}+\kappa(n) s+\lambda(n), \quad s \in \mathbf{R} .
$$

Подставим представление (33) для функции $f_{n}(s)$ в $(30)$, полагая $h=$ $(0, m), k=(t, 0)$. Получаем

$$
\begin{aligned}
\Delta_{k}^{2} \Delta_{h} f_{n}(s) & =\Delta_{h} \Delta_{t}^{2} f_{n}(s)=\Delta_{h} \sigma(n) \Delta_{t}^{2} s^{2} \\
& =2 t^{2} \Delta_{m} \sigma(n)=0, \quad t \in \mathbf{R}, \quad m \in \mathbf{Z} .
\end{aligned}
$$

Отсюда следует, что $\Delta_{m} \sigma(n)=0$, т.е. $\sigma(n)=\sigma=$ const.

Так как $f(-y)=f(y), y \in Y$, то очевидно, что $\kappa(-n)=-\kappa(n)$ и $\lambda(-n)=\lambda(n), n \in \mathbf{Z}$. Отметим также, что любая функция $f(s, n)$, имеющая вид (31), удовлетворяет уравнению (30). Лемма доказана.

4. Доказательство теоремы 1. Пусть $X$ - произвольная локально компактная абелева группа, удовлетворяющая второй аксиоме счетности. Заметим вначале, что если $\mu$ - распределение случайной величины $\xi$ со значениями в группе $X$ и $\alpha \in \operatorname{Aut}(X)$, то характеристическая функция случайной величины $\alpha \xi$ равна $\widehat{\mu}(\widetilde{\alpha} y)$. Отсюда вытекает, что $\mu \in \Gamma(X)$ тогда и только тогда, когда $\alpha(\mu) \in \Gamma(X)$. Поэтому, полагая $\zeta_{j}=\alpha_{1 j} \xi_{j}, j=1,2,3$, мы сводим доказательство теоремы 1 к случаю, когда линейные статистики имеют вид $L_{1}=\xi_{1}+\xi_{2}+\xi_{3}$, $L_{2}=\delta_{21} \xi_{1}+\delta_{22} \xi_{2}+\delta_{23} \xi_{3}, L_{3}=\delta_{31} \xi_{1}+\delta_{32} \xi_{2}+\delta_{33} \xi_{3}$, где $\delta_{i j} \in \operatorname{Aut}(X)$. Заметим также, что если $\alpha, \beta \in \operatorname{Aut}(X)$, то линейные статистики $L_{1}$, $L_{2}$ и $L_{3}$ независимы тогда и только тогда, когда независимы линейные статистики $L_{1}, \alpha L_{2}$ и $\beta L_{3}$. Поэтому, доказывая теорему, можно предполагать, что $L_{1}=\xi_{1}+\xi_{2}+\xi_{3}, L_{2}=\alpha_{1} \xi_{1}+\alpha_{2} \xi_{2}+\xi_{3}, L_{3}=\beta_{1} \xi_{1}+\beta_{2} \xi_{2}+\xi_{3}$, где $\alpha_{i}=\left(\begin{array}{cc}a_{i} & c_{i} \\ 0 & p_{i}\end{array}\right), \beta_{i}=\left(\begin{array}{cc}b_{i} & d_{i} \\ 0 & q_{i}\end{array}\right), i=1,2$, - топологические автоморфизмы группы $X=\mathbf{R} \times \mathbf{T}$.

Положим $\nu_{j}=\mu_{j} * \bar{\mu}_{j}$. Учитывая лемму 7 , мы можем доказывать теорему, предполагая, что все распределения $\mu_{j}$ невырожденные. Более того, как следует из доказательства леммы 7, в этом случае $\widehat{\nu}_{j}(s, 0)=e^{-\sigma_{j} s^{2}}$, где $s \in \mathbf{R}, \sigma_{j}>0, j=1,2,3$. Отметим, что тогда $\sigma_{j}$ удовлетворяют уравнениям (6)-(8) и выполнены утверждения 1)-5) леммы 2.

По лемме 1 характеристические функции $\widehat{\mu}_{j}(y)$ удовлетворяют уравнению (2). Очевидно, что характеристические функции $\widehat{\nu}_{j}(y)$ также удовлетворяют уравнению (2). Положим $\psi_{j}(y)=-\ln \widehat{\nu}_{j}(y)$. Тогда из $(2)$ следует, что функции $\psi_{j}(y)$ удовлетворяют уравнению $(21)$.

По лемме 6 функция $\psi_{1}(s, n)$ удовлетворяет уравнению (18). Воспользуемся теперь леммой 7 и заметим, что всегда выполнено включение

$$
\mathbf{R} \subset N \cap L .
$$


Из (18) и (34) тогда следует, что функция $\psi_{1}(s, n)$ удовлетворяет уравнению (30). Рассуждая аналогично, получаем, что функции $\psi_{2}(s, n)$ и $\psi_{3}(s, n)$ также удовлетворяют уравнению (30). Применяя к функциям $\psi_{j}(s, n)$ лемму 8, получаем, что имеют место представления

$$
\psi_{j}(s, n)=\sigma_{j} s^{2}+\kappa_{j}(n) s+\lambda_{j}(n), \quad s \in \mathbf{R}, \quad n \in \mathbf{Z},
$$

где $\kappa_{j}(-n)=-\kappa_{j}(n), \lambda_{j}(-n)=\lambda_{j}(n)$ и $\kappa_{j}(0)=0, \lambda_{j}(0)=0, j=1,2,3$.

По лемме $6, \nu=\nu_{1} * \nu_{2} * \nu_{3} \in \Gamma(X)$. Тогда очевидно, что

$$
\widehat{\nu}(s, n)=\exp \left\{-\left(\sigma s^{2}+\kappa s n+\lambda n^{2}\right)\right\}, \quad s \in \mathbf{R}, \quad n \in \mathbf{Z},
$$

где $\sigma>0, \kappa \in \mathbf{R}, \lambda \geqslant 0$ и

$$
\psi_{1}(s, n)+\psi_{2}(s, n)+\psi_{3}(s, n)=\sigma s^{2}+\kappa s n+\lambda n^{2}, \quad s \in \mathbf{R}, \quad n \in \mathbf{Z}
$$

Проверим, что функции $\kappa_{j}(n)$ в $(35)$ линейные. Положим в $(21)$ $u=(0, n), v=\left(s_{2}, 0\right), w=\left(s_{3}, 0\right)$. Отметим, что $\widetilde{\alpha}_{1} v=\left(a_{1} s_{2}, 0\right), \widetilde{\alpha}_{2} v=$ $\left(a_{2} s_{2}, 0\right), \widetilde{\beta}_{1} w=\left(b_{1} s_{3}, 0\right), \widetilde{\beta}_{2} w=\left(b_{2} s_{3}, 0\right)$. Учитывая представления (35), из (21) находим

$$
\begin{aligned}
\sigma_{1}( & \left.a_{1} s_{2}+b_{1} s_{3}\right)^{2}+\kappa_{1}(n)\left(a_{1} s_{2}+b_{1} s_{3}\right)+\lambda_{1}(n)+\sigma_{2}\left(a_{2} s_{2}+b_{2} s_{3}\right)^{2} \\
& \quad+\kappa_{2}(n)\left(a_{2} s_{2}+b_{2} s_{3}\right)+\lambda_{2}(n)+\sigma_{3}\left(s_{2}+s_{3}\right)^{2}+\kappa_{3}(n)\left(s_{2}+s_{3}\right)+\lambda_{3}(n) \\
= & \lambda_{1}(n)+\lambda_{2}(n)+\lambda_{3}(n)+\sigma_{1}\left(a_{1} s_{2}\right)^{2}+\sigma_{2}\left(a_{2} s_{2}\right)^{2}+\sigma_{3} s_{2}^{2} \\
& +\sigma_{1}\left(b_{1} s_{3}\right)^{2}+\sigma_{2}\left(b_{2} s_{3}\right)^{2}+\sigma_{3} s_{3}^{2}, \quad s_{2}, s_{3} \in \mathbf{R}, \quad n \in \mathbf{Z} .
\end{aligned}
$$

Полагая в $(38) s_{2}=1, s_{3}=0$, получаем

$$
a_{1} \kappa_{1}(n)+a_{2} \kappa_{2}(n)+\kappa_{3}(n)=0, \quad n \in \mathbf{Z} .
$$

Полагая в $(38) s_{2}=0, s_{3}=1$, находим

$$
b_{1} \kappa_{1}(n)+b_{2} \kappa_{2}(n)+\kappa_{3}(n)=0, \quad n \in \mathbf{Z} .
$$

С другой стороны, из (35) и (37) следует, что

$$
\kappa_{1}(n)+\kappa_{2}(n)+\kappa_{3}(n)=\kappa n, \quad n \in \mathbf{Z} .
$$

Вычитая из (39) и (40) равенство (41), получаем

$$
\begin{aligned}
& \left(a_{1}-1\right) \kappa_{1}(n)+\left(a_{2}-1\right) \kappa_{2}(n)=-\kappa n, \quad n \in \mathbf{Z}, \\
& \left(b_{1}-1\right) \kappa_{1}(n)+\left(b_{2}-1\right) \kappa_{2}(n)=-\kappa n, \quad n \in \mathbf{Z} \text {. }
\end{aligned}
$$

Так как согласно утверждению 5) леммы 2 справедливо неравенство $\left|\begin{array}{ll}a_{1}-1 & a_{2}-1 \\ b_{1}-1 & b_{2}-1\end{array}\right| \neq 0$, то из уравнений $(41)-(43)$ следует, что функции $\kappa_{j}(n)$ 
линейные, т.е. $\kappa_{j}(n)=\kappa_{j} n, n \in \mathbf{Z}$, где $\kappa_{j} \in \mathbf{R}$. Таким образом, представления (35) принимают вид

$$
\psi_{j}(s, n)=\sigma_{j} s^{2}+\kappa_{j} s n+\lambda_{j}(n), \quad s \in \mathbf{R}, \quad n \in \mathbf{Z}, \quad j=1,2,3 .
$$

Отметим, что из (37) и (44) следует также, что

$$
\lambda_{1}(n)+\lambda_{2}(n)+\lambda_{3}(n)=\lambda n^{2}, \quad n \in \mathbf{Z} .
$$

Пусть $u=\left(s_{1}, n_{1}\right), v=\left(s_{2}, n_{2}\right), w=\left(s_{3}, n_{3}\right)$. Тогда

$$
\begin{array}{ll}
\widetilde{\alpha}_{1} v=\left(a_{1} s_{2}+c_{1} n_{2}, p_{1} n_{2}\right), & \widetilde{\alpha}_{2} v=\left(a_{2} s_{2}+c_{2} n_{2}, p_{2} n_{2}\right), \\
\widetilde{\beta}_{1} w=\left(b_{1} s_{3}+d_{1} n_{3}, q_{1} n_{3}\right), & \widetilde{\beta}_{2} w=\left(b_{2} s_{3}+d_{2} n_{3}, q_{2} n_{3}\right) .
\end{array}
$$

Подставляя представления (44) в (21), получаем, что справедливы равенства (6)-(8), а также равенства

$$
\begin{aligned}
& \kappa_{1} a_{1}+\kappa_{2} a_{2}+\kappa_{3}=0 \\
& \kappa_{1} b_{1}+\kappa_{2} b_{2}+\kappa_{3}=0 \\
& 2 \sigma_{1} c_{1}+2 \sigma_{2} c_{2}+\kappa_{1} p_{1}+\kappa_{2} p_{2}+\kappa_{3}=0 \\
& 2 \sigma_{1} d_{1}+2 \sigma_{2} d_{2}+\kappa_{1} q_{1}+\kappa_{2} q_{2}+\kappa_{3}=0 \\
& 2 \sigma_{1} a_{1} d_{1}+2 \sigma_{2} a_{2} d_{2}+\kappa_{1} a_{1} q_{1}+\kappa_{2} a_{2} q_{2}+\kappa_{3}=0 \\
& 2 \sigma_{1} b_{1} c_{1}+2 \sigma_{2} b_{2} c_{2}+\kappa_{1} b_{1} p_{1}+\kappa_{2} b_{2} p_{2}+\kappa_{3}=0 \\
& n_{1} n_{2}\left(\kappa_{1} c_{1}+\kappa_{2} c_{2}\right)+n_{1} n_{3}\left(\kappa_{1} d_{1}+\kappa_{2} d_{2}\right) \\
& \quad+n_{2} n_{3}\left(2 \sigma_{1} c_{1} d_{1}+2 \sigma_{2} c_{2} d_{2}+\kappa_{1} c_{1} q_{1}+\kappa_{1} d_{1} p_{1}+\kappa_{2} c_{2} q_{2}+\kappa_{2} d_{2} p_{2}\right) \\
& \quad+\lambda_{1}\left(n_{1}+p_{1} n_{2}+q_{1} n_{3}\right)+\lambda_{2}\left(n_{1}+p_{2} n_{2}+q_{2} n_{3}\right) \\
& \quad+\lambda_{3}\left(n_{1}+n_{2}+n_{3}\right)=\lambda\left(n_{1}^{2}+n_{2}^{2}+n_{3}^{2}\right),
\end{aligned}
$$

Заметим, что из (37) и (44) следует, что

$$
\sigma_{1}+\sigma_{2}+\sigma_{3}=\sigma
$$

и

$$
\kappa_{1}+\kappa_{2}+\kappa_{3}=\kappa
$$

Докажем, что носителем распределения $\nu$ является некоторая подгруппа в $X$, топологически изоморфная $\mathbf{R}$. Учитывая $(36)$, для этого достаточно проверить, что

$$
4 \lambda \sigma=\kappa^{2}
$$

т.е., с учетом (53) и (54), нужно доказать, что

$$
4 \lambda\left(\sigma_{1}+\sigma_{2}+\sigma_{3}\right)=\left(\kappa_{1}+\kappa_{2}+\kappa_{3}\right)^{2} .
$$


Проверка (56) - это ряд элементарных и скучных вычислений. Учитывая, что согласно утверждению 4) леммы 2 справедливо неравенство $a_{2} b_{1}-a_{1} b_{2} \neq 0$, из (6) и (7) получаем

$$
\sigma_{1}=\frac{b_{2}-a_{2}}{a_{2} b_{1}-a_{1} b_{2}} \sigma_{3}, \quad \sigma_{2}=\frac{a_{1}-b_{1}}{a_{2} b_{1}-a_{1} b_{2}} \sigma_{3} .
$$

Аналогично, из (46) и (47) находим

$$
\kappa_{1}=\frac{b_{2}-a_{2}}{a_{2} b_{1}-a_{1} b_{2}} \kappa_{3}, \quad \kappa_{2}=\frac{a_{1}-b_{1}}{a_{2} b_{1}-a_{1} b_{2}} \kappa_{3} .
$$

Каждое из чисел $p_{j}$ и $q_{j}$ может принимать значения \pm 1 . Здесь возможны несколько случаев, каждый из которых мы разберем отдельно.

I. Пусть $p_{1}=p_{2}=1$. Тогда из (48) следует, что

$$
\kappa_{1}+\kappa_{2}+\kappa_{3}=-2 \sigma_{1} c_{1}-2 \sigma_{2} c_{2} .
$$

Полагая в $(52) n_{1}=-n_{2}=1, n_{3}=0$, получаем

$$
-\kappa_{1} c_{1}-\kappa_{2} c_{2}=2 \lambda \text {. }
$$

Подставляя (59) и (60) в (56) и учитывая (57) и (58), убеждаемся, что мы получили равенство.

Таким образом, в случае I равенство (55) доказано.

II. Пусть $q_{1}=q_{2}=1$. Тогда из (49) следует, что

$$
\kappa_{1}+\kappa_{2}+\kappa_{3}=-2 \sigma_{1} d_{1}-2 \sigma_{2} d_{2} .
$$

Полагая в (52) $n_{1}=-n_{3}=1, n_{2}=0$, получаем

$$
-\kappa_{1} d_{1}-\kappa_{2} d_{2}=2 \lambda .
$$

Подставляя (61) и (62) в (56) и учитывая (57) и (58), убеждаемся, что мы получили равенство.

Таким образом, в случае II равенство (55) доказано.

III. Пусть либо $p_{1}=p_{2}=q_{1}=q_{2}=-1$, либо $p_{1}=q_{1}=-1, p_{2}=q_{2}=$ 1 , либо $p_{1}=q_{1}=1, p_{2}=q_{2}=-1$. Полагая в (52) $n_{1}=0, n_{2}=-n_{3}=1$, получаем

$$
-\left(2 \sigma_{1} c_{1} d_{1}+2 \sigma_{2} c_{2} d_{2}+\kappa_{1} c_{1} p_{1}+\kappa_{1} d_{1} p_{1}+\kappa_{2} c_{2} p_{2}+\kappa_{2} d_{2} p_{2}\right)=2 \lambda .
$$

Заметим, что из утверждения 3 ) леммы 2 следует, что $a_{1} \neq a_{2}$ и $b_{1} \neq b_{2}$. Из (48) и (51) находим

$$
c_{1}=\frac{\left(1-b_{2}\right) \kappa_{3}-\kappa_{1} p_{1}\left(b_{2}-b_{1}\right)}{2 \sigma_{1}\left(b_{2}-b_{1}\right)}, \quad c_{2}=\frac{\left(1-b_{1}\right) \kappa_{3}-\kappa_{2} p_{2}\left(b_{1}-b_{2}\right)}{2 \sigma_{2}\left(b_{1}-b_{2}\right)} .
$$


Из (49) и (50) получаем

$$
d_{1}=\frac{\left(1-a_{2}\right) \kappa_{3}-\kappa_{1} q_{1}\left(a_{2}-a_{1}\right)}{2 \sigma_{1}\left(a_{2}-a_{1}\right)}, \quad d_{2}=\frac{\left(1-a_{1}\right) \kappa_{3}-\kappa_{2} q_{2}\left(a_{1}-a_{2}\right)}{2 \sigma_{2}\left(a_{1}-a_{2}\right)} .
$$

Находя из (63) $\lambda$ и подставляя полученное выражение в (56), видим, что нужно проверить равенство

$$
\begin{gathered}
-2\left(2 \sigma_{1} c_{1} d_{1}+2 \sigma_{2} c_{2} d_{2}+\kappa_{1} c_{1} p_{1}+\kappa_{1} d_{1} p_{1}+\kappa_{2} c_{2} p_{2}+\kappa_{2} d_{2} p_{2}\right) \\
\times\left(\sigma_{1}+\sigma_{2}+\sigma_{3}\right)=\left(\kappa_{1}+\kappa_{2}+\kappa_{3}\right)^{2} .
\end{gathered}
$$

Подставим (57), (58), (64) и (65) в (66). После элементарных преобразований убеждаемся в том, что проверка (66) сводится к проверке равенства

$$
\begin{gathered}
\left(a_{2} b_{1}-a_{1} b_{2}\right)\left(\left(1-b_{2}\right)\left(1-a_{2}\right)\left(a_{1}-b_{1}\right)+\left(1-b_{1}\right)\left(1-a_{1}\right)\left(b_{2}-a_{2}\right)\right) \\
=-\left(b_{2}-b_{1}\right)\left(a_{2}-a_{1}\right)\left(a_{1}-b_{1}\right)\left(b_{2}-a_{2}\right) .
\end{gathered}
$$

Справедливость (67) вытекает из утверждения 1) леммы 2.

Таким образом, в случае III равенство (55) доказано.

Для рассмотрения оставшихся случаев, положим в (52) $n_{1}=n_{2}=$ $n_{3}=1$. Принимая во внимание, что $\lambda_{j}(-n)=\lambda_{j}(n), n \in \mathbf{Z}, j=1,2$, мы получаем

$$
\begin{aligned}
\kappa_{1} c_{1}+\kappa_{2} c_{2}+\kappa_{1} d_{1}+\kappa_{2} d_{2}+2 \sigma_{1} c_{1} d_{1}+2 \sigma_{2} c_{2} d_{2}+\kappa_{1} c_{1} q_{1} \\
+\kappa_{1} d_{1} p_{1}+\kappa_{2} c_{2} q_{2}+\kappa_{2} d_{2} p_{2}+\lambda_{1}(1)+\lambda_{2}(1)+\lambda_{3}(3)=3 \lambda .
\end{aligned}
$$

IV. Пусть $p_{1}=p_{2}=-1$ и $q_{1}=-q_{2}$. Полагая в $(52) n_{1}=2, n_{2}=$ $1, n_{3}=0$, получаем

$$
2\left(\kappa_{1} c_{1}+\kappa_{2} c_{2}\right)+\lambda_{1}(1)+\lambda_{2}(1)+\lambda_{3}(3)=5 \lambda .
$$

Отсюда и из (68) находим

$$
\kappa_{1} c_{1}+\kappa_{2} c_{2}-\left(2 \sigma_{1} c_{1} d_{1}+2 \sigma_{2} c_{2} d_{2}+\kappa_{1} c_{1} q_{1}-\kappa_{2} c_{2} q_{1}\right)=2 \lambda
$$

$\mathrm{V}$. Пусть $q_{1}=q_{2}=-1$ и $p_{1}=-p_{2}$. Полагая в $(52) n_{1}=2, n_{2}=0$, $n_{3}=1$, получаем

$$
2\left(\kappa_{1} d_{1}+\kappa_{2} d_{2}\right)+\lambda_{1}(1)+\lambda_{2}(1)+\lambda_{3}(3)=5 \lambda
$$

Отсюда и из (68) находим

$$
\kappa_{1} d_{1}+\kappa_{2} d_{2}-\left(2 \sigma_{1} c_{1} d_{1}+2 \sigma_{2} c_{2} d_{2}+\kappa_{1} d_{1} p_{1}-\kappa_{2} d_{2} p_{1}\right)=2 \lambda
$$


VI. Пусть либо $p_{1}=q_{2}=-1, p_{2}=q_{1}=1$, либо $p_{1}=q_{2}=1$, $p_{2}=q_{1}=-1$. Полагая в $(52) n_{1}=0, n_{2}=2, n_{3}=1$, получаем

$$
\begin{gathered}
2\left(2 \sigma_{1} c_{1} d_{1}+2 \sigma_{2} c_{2} d_{2}+\kappa_{1} c_{1} q_{1}+\kappa_{1} d_{1} p_{1}+\kappa_{2} c_{2} q_{2}+\kappa_{2} d_{2} p_{2}\right) \\
+\lambda_{1}(1)+\lambda_{2}(1)+\lambda_{3}(3)=5 \lambda .
\end{gathered}
$$

Отсюда и из (68) находим

$$
\begin{aligned}
2 \sigma_{1} c_{1} d_{1} & +2 \sigma_{2} c_{2} d_{2}+\kappa_{1} c_{1} p_{2}+\kappa_{1} d_{1} p_{1}+\kappa_{2} c_{2} p_{1}+\kappa_{2} d_{2} p_{2} \\
& -\left(\kappa_{1} d_{1}+\kappa_{2} d_{2}\right)-\left(\kappa_{1} c_{1}+\kappa_{2} c_{2}\right)=2 \lambda .
\end{aligned}
$$

Далее в каждом из этих случаев рассуждаем так же, как в случае III. Итак, мы доказали, что справедливо равенство (55). Таким образом, получаем представление

$$
\begin{aligned}
\widehat{\nu}(s, n) & =\exp \left\{-\left(\sigma s^{2}+\kappa s n+\lambda n^{2}\right)\right\} \\
& =\exp \left\{-\sigma\left(s+\frac{\kappa}{2 \sigma} n\right)^{2}\right\}, \quad s \in \mathbf{R}, \quad n \in \mathbf{Z} .
\end{aligned}
$$

Положим $H=\{y \in Y: \widehat{\nu}(y)=1\}$. Очевидно, что $H=$ $\{n(-\kappa /(2 \sigma), 1): n \in \mathbf{Z}\}$. Тогда $G=A(X, H)=\left\{\left(t, e^{i t \kappa /(2 \sigma)}\right): t \in \mathbf{R}\right\}$. Очевидно, что $G \cong \mathbf{R}$. Мы имеем $\sigma(\nu) \subset G$. Поскольку $\nu=\nu_{1} * \nu_{2} * \nu_{3}=$ $\mu_{1} * \bar{\mu}_{1} * \mu_{2} * \bar{\mu}_{2} * \mu_{3} * \bar{\mu}_{3}$, то из леммы 3 следует, что распределения $\mu_{j}$ можно так заменить их сдвигами $\mu_{j}^{\prime}$, что $\nu=\mu_{1}^{\prime} * \bar{\mu}_{1}^{\prime} * \mu_{2}^{\prime} * \bar{\mu}_{2}^{\prime} * \mu_{3}^{\prime} * \bar{\mu}_{3}^{\prime}$, а $\sigma\left(\mu_{j}^{\prime}\right) \subset G$. Так как $\nu$ - гауссовское распределение, а $G \cong \mathbf{R}$, то по теореме Крамера о разложении гауссовского распределения получаем, что все распределения $\mu_{j}^{\prime}$ - гауссовские, а значит, $\mu_{j}$ - также гауссовские. Теорема доказана.

\section{5. Комментарии к теореме 1.}

3 а м е ч а н и е 1 . При $n=2$ на группе $X=\mathbf{R} \times \mathbf{T}$ в условиях задачи 1 распределения $\mu_{j}$ являются либо гауссовскими, либо свертками гауссовских распределений с зарядами, сосредоточенными на $\mathbf{Z}(2)$ [15]. Согласно теореме 1 , при $n=3$ распределения $\mu_{j}$ - только гауссовские. Мы видим, что, в отличие от вещественной прямой $\mathbf{R}$, на группе $X=\mathbf{R} \times \mathbf{T}$ класс распределений, характеризующийся независимостью $n$ линейных статистик от $n$ независимых случайных величин, зависит от $n$.

3 а м е ч а н и е 2. Как установлено при доказательстве теоремы 1 , если линейные статистики $L_{i}$ имеют вид $L_{1}=\xi_{1}+\xi_{2}+\xi_{3}, L_{2}=\alpha_{1} \xi_{1}+$ $\alpha_{2} \xi_{2}+\xi_{3}, L_{3}=\beta_{1} \xi_{1}+\beta_{2} \xi_{2}+\xi_{3}$ и распределения $\mu_{j}$ невырожденные, то носители $\mu_{j}$ - классы смежности группы $X$ относительно одной и той же однопараметрической подгруппы $G=\left\{\left(t, e^{i t \kappa /(2 \sigma)}\right): t \in \mathbf{R}\right\}$. 
Проверим, что подгруппа $G$ инвариантна относительно всех автоморфизмов $\alpha_{i}, \beta_{i}$. Учитывая (53), (54), (57) и (58), получаем, что $G=\left\{\left(t, e^{i t \kappa_{3} /\left(2 \sigma_{3}\right)}\right): t \in \mathbf{R}\right\}$. Проверим, что подгруппа $G$ инвариантна относительно автоморфизма $\alpha_{1}$. Так как

$$
\begin{aligned}
\alpha_{1}\left(t, \exp \left\{i t \frac{\kappa_{3}}{2 \sigma_{3}}\right\}\right) & =\left(a_{1} t, \exp \left\{i c_{1} t\right\} \exp \left\{i t p_{1} \frac{\kappa_{3}}{2 \sigma_{3}}\right\}\right) \\
& =\left(a_{1} t, \exp \left\{i a_{1} t \frac{\kappa_{3}}{2 \sigma_{3}}\left(\frac{2 c_{1} \sigma_{3}}{a_{1} \kappa_{3}}+\frac{p_{1}}{a_{1}}\right)\right\}\right)
\end{aligned}
$$

то для этого достаточно показать, что

$$
\frac{2 c_{1} \sigma_{3}}{a_{1} \kappa_{3}}+\frac{p_{1}}{a_{1}}=1
$$

Для проверки равенства (69) вначале подставим в (69) представление для $c_{1}$ из (64), а затем в полученное выражение подставим представления для $\sigma_{1}$ и $\kappa_{1}$ из $(57)$ и (58) соответственно. После простых преобразований убеждаемся, что проверяемое равенство сводится к равенству 1) в лемме 2.

Аналогично проверяем, что подгруппа $G$ инвариантна относительно автоморфизмов $\alpha_{2}, \beta_{1}, \beta_{2}$. Очевидно, что сужение каждого из автоморфизмов $\alpha_{i}, \beta_{i}$ на группу $G$ является топологическим автоморфизмом $G$.

Из сказанного следует, что вывод о том, что распределения $\mu_{j}$ в теореме 1 - гауссовские, можно сделать не используя теорему Крамера, а непосредственно из теоремы Скитовича-Дармуа для вещественной прямой.

Отметим также, что условие инвариантности $G$ относительно автоморфизмов $\alpha_{i}, \beta_{i}$ накладывает достаточно жесткие ограничения на $\alpha_{i}, \beta_{i}$. Действительно, пусть $G$ - произвольная подгруппа в группе $X=\mathbf{R} \times \mathbf{T}$, топологически изоморфная $\mathbf{R}$. Тогда $G$ имеет вид $G=\left\{\left(t, e^{i \omega t}\right): t \in \mathbf{R}\right\}$, где $\omega$ - фиксированное вещественное число. Очевидно, что подгруппа $G$ инвариантна относительно автоморфизма $\alpha=\left(\begin{array}{ll}a & c \\ 0 & p\end{array}\right) \in \operatorname{Aut}(X)$ тогда и только тогда, когда $c=(a-p) \omega$.

Пусть $\omega=0$, т.е. $G=\{(t, 1): t \in \mathbf{R}\}$. Тогда $c_{1}=c_{2}=d_{1}=d_{2}=0$, т.е. матрицы автоморфизмов $\alpha_{i}, \beta_{i}$ диагональные, причем для $a_{i}$ и $b_{i}$ выполнены утверждения 1)-5) леммы 2.

Пусть $\omega \neq 0$. Предположим также, что ни один из автоморфизмов $\alpha_{i}, \beta_{i}$ не равен $\pm I$. Тогда, помимо выполнения утверждений 1) -5 ) леммы 2 , должны быть справедливы равенства

$$
\frac{c_{1}}{a_{1}-p_{1}}=\frac{c_{2}}{a_{2}-p_{2}}=\frac{d_{1}}{b_{1}-q_{1}}=\frac{d_{2}}{b_{2}-q_{2}} .
$$

В частности, если хотя бы одно из этих равенств не выполнено, то распределения $\mu_{j}$ вырожденные. Если же какие-либо из автоморфизмов 
$\alpha_{i}, \beta_{i}$ равны $\pm I$, то в (70) отсутствуют выражения, отвечающие соответствующим автоморфизмам.

3 а м е ч а н и е 3 . Теорема 1 не может быть усилена до утверждения, что $\mu_{j}$ - вырожденные распределения (ср. с леммой 5). Действительно, пусть $G$ - произвольная однопараметрическая подгруппа в группе $X=\mathbf{R} \times \mathbf{T}$, имеющая вид $G=\left\{\left(t, e^{i \omega t}\right): t \in \mathbf{R}\right\}$, где $\omega-$ фиксированное вещественное число. Пусть ненулевые вещественные числа $a_{i}, b_{i}$ таковы, что система уравнений (6)-(8) имеет решение $\sigma_{1}>0, \sigma_{2}>0$, $\sigma_{3}>0$. Рассмотрим автоморфизмы $\alpha_{i}, \beta_{i} \in \operatorname{Aut}(X), i=1,2$, вида

$$
\begin{array}{ccc}
\alpha_{1}=\left(\begin{array}{cc}
a_{1} & \left(a_{1}-p_{1}\right) \omega \\
0 & p_{1}
\end{array}\right), \quad \alpha_{2}=\left(\begin{array}{cc}
a_{2} & \left(a_{2}-p_{2}\right) \omega \\
0 & p_{2}
\end{array}\right), \\
\beta_{1}=\left(\begin{array}{cc}
b_{1} & \left(b_{1}-q_{1}\right) \omega \\
0 & q_{1}
\end{array}\right), & \beta_{2}=\left(\begin{array}{cc}
b_{2} & \left(b_{2}-q_{2}\right) \omega \\
0 & q_{2}
\end{array}\right) .
\end{array}
$$

Пусть $\xi_{j}, j=1,2,3$, - независимые гауссовские случайные величины со значениями в группе $X$ и с распределениями $\mu_{j}$, имеющими характеристические функции

$$
\begin{gathered}
\widehat{\mu}_{1}(s, n)=\exp \left\{-\sigma_{1}(s+\omega n)^{2}\right\}, \quad \widehat{\mu}_{2}(s, n)=\exp \left\{-\sigma_{2}(s+\omega n)^{2}\right\}, \\
\widehat{\mu}_{3}(s, n)=\exp \left\{-\sigma_{3}(s+\omega n)^{2}\right\}, \quad s \in \mathbf{R}, \quad n \in \mathbf{Z} .
\end{gathered}
$$

Очевидно, что носители всех распределений $\mu_{j}$ совпадают с подгруппой $G$. Учитывая (6)-(8), легко непосредственно проверить, что характеристические функции $\widehat{\mu}_{j}(s, n)$ удовлетворяют уравнению $(2)$, а следовательно, по лемме 1 линейные статистики $L_{1}=\xi_{1}+\xi_{2}+\xi_{3}, L_{2}=$ $\alpha_{1} \xi_{1}+\alpha_{2} \xi_{2}+\xi_{3}, L_{3}=\beta_{1} \xi_{1}+\beta_{2} \xi_{2}+\xi_{3}$ независимы.

3 а м е ч а н и е 4 . На группе $X=\mathbf{T}$ при $n=2$ в условиях задачи 1 распределения $\mu_{j}$ являются либо гауссовскими, либо свертками гауссовских распределений с зарядами [18], а при $n=3$ все распределения $\mu_{j}$ по лемме 5 - вырожденные. На группе $X=\mathbf{R} \times \mathbf{T}$ при $n=2$ в условиях задачи 1 распределения $\mu_{j}$ также являются либо гауссовскими, либо свертками гауссовских распределений с зарядами [15], а при $n=3$ все распределения $\mu_{j}$ по теореме 1 - гауссовские. Может возникнуть предположение, что с увеличением $n$ класс распределений в задаче 1 , характеризующийся независимостью $n$ линейных статистик от $n$ независимых случайных величин, сужается. Оказывается, что это предположение не верно.

Действительно, пусть $\xi_{1}, \xi_{2}, \xi_{3}, \xi_{4}$ - независимые случайные величины со значениями в $\mathbf{T}$ и распределениями $\mu_{j}$. Рассмотрим линейные статистики $L_{1}=\xi_{1}+\xi_{2}+\xi_{3}+\xi_{4}, L_{2}=\xi_{1}+\xi_{2}-\xi_{3}-\xi_{4}, L_{3}=\xi_{1}-\xi_{2}+\xi_{3}-\xi_{4}$, $L_{4}=\xi_{1}-\xi_{2}-\xi_{3}+\xi_{4}$. Пусть $\mu_{1}=\mu_{2}=\gamma * \pi_{1}, \mu_{3}=\mu_{4}=\gamma * \pi_{2}$, где $\gamma \in \Gamma(\mathbf{T})$, а $\pi_{j}$ - сосредоточенные на $\mathbf{Z}(2)$ заряды такие, что $\pi_{1} * \pi_{2}=E_{1}$. Легко проверить, что в этом случае линейные статистики $L_{1}, L_{2}, L_{3}, L_{4}$ независимы, но ни одно $\mu_{j}$ не принадлежит $\Gamma(\mathbf{T})$. 
6. Решение задачи 1 на группе $X=\Sigma_{\boldsymbol{a}} \times \mathbf{T}$. Перейдем теперь решению задачи 1 в случае $n=3$ на группе $X=\Sigma_{\boldsymbol{a}} \times \mathbf{T}$. Напомним определение $\boldsymbol{a}$-адического соленоида $\Sigma_{\boldsymbol{a}}$.

Пусть $\boldsymbol{a}=\left(a_{0}, a_{1}, \ldots\right)$, где $a_{j} \in \mathbf{Z}, a_{j}>1, j=0,1, \ldots$ Вначале мы определим группу целых $\boldsymbol{a}$-адических чисел $\boldsymbol{\Delta}_{\boldsymbol{a}}$. Как множество $\Delta_{\boldsymbol{a}}$ совпадает с декартовым произведением $\underset{n=0}{\mathbf{P}}\left\{0,1, \ldots, a_{n}-1\right\}$. Рассмотрим $\boldsymbol{x}=\left(x_{0}, x_{1}, x_{2}, \ldots\right), \boldsymbol{y}=\left(y_{0}, y_{1}, y_{2}, \ldots\right) \in \Delta_{\boldsymbol{a}}$ и определим сумму $\boldsymbol{z}=\boldsymbol{x}+\boldsymbol{y}$ следующим образом. Пусть $x_{0}+y_{0}=t_{0} a_{0}+z_{0}$, где $z_{0} \in\left\{0,1, \ldots, a_{0}-1\right\}$, $t_{0} \in\{0,1\}$. Предположим, что числа $z_{0}, z_{1}, \ldots, z_{k}$ и $t_{0}, t_{1}, \ldots, t_{k}$ уже определены. Положим тогда $x_{k+1}+y_{k+1}+t_{k}=t_{k+1} a_{k+1}+z_{k+1}$, где $z_{k+1} \in\left\{0,1, \ldots, a_{k+1}-1\right\}, t_{k+1} \in\{0,1\}$. Таким образом, по индукции определена последовательность $\boldsymbol{z}=\left(z_{0}, z_{1}, z_{2}, \ldots\right)$. Множество $\Delta_{\boldsymbol{a}}$ с определенным выше сложением является абелевой группой. Эта группа, рассматриваемая в топологии произведения, называется группой целых $\boldsymbol{a}$-адических чисел. Рассмотрим группу $\mathbf{R} \times \Delta_{\boldsymbol{a}}$. Пусть $B-$ подгруппа в $\mathbf{R} \times \Delta_{\boldsymbol{a}}$ вида $B=\{(n, n \boldsymbol{u})\}_{n=-\infty}^{\infty}$, где $\boldsymbol{u}=(1,0, \ldots, 0, \ldots)$. Факторгруппа $\Sigma_{\boldsymbol{a}}=\left(\mathbf{R} \times \Delta_{\mathbf{a}}\right) / B$ называется $\boldsymbol{a}$-адическим соленоидом. Группа $\Sigma_{\boldsymbol{a}}$ компактна, связна и имеет размерность один $[24$, формулы $(10.12)$, (10.13), (24.28)]. Пусть $\mathbf{Q}$ обозначает группу рациональных чисел, рассматриваемую в дискретной топологии. Группа характеров группы $\Sigma_{\boldsymbol{a}}$ топологически изоморфна подгруппе $H_{\boldsymbol{a}} \subset \mathbf{Q}$ вида

$$
H_{\boldsymbol{a}}=\left\{\frac{m}{a_{0} a_{1} \cdots a_{n}}: n=0,1, \ldots ; m \in \mathbf{Z}\right\}
$$

$[24, \S 25.3]$.

Пусть $X=\Sigma_{\boldsymbol{a}} \times \mathbf{T}$. Тогда $Y \cong H_{\boldsymbol{a}} \times \mathbf{Z}$. Чтобы не усложнять обозначения, будем считать, что $Y=H_{\boldsymbol{a}} \times \mathbf{Z}$. Элементы группы $X$ будем обозначать через $x=(g, z), g \in \Sigma_{\boldsymbol{a}}, z \in \mathbf{T}$, а элементы группы $Y$ - через $y=(r, n), r \in H_{\boldsymbol{a}}, n \in \mathbf{Z}$. Нетрудно проверить, что каждый автоморфизм $\varepsilon \in \operatorname{Aut}(Y)$ определяется матрицей $\left(\begin{array}{ll}a & c \\ 0 & p\end{array}\right)$, где $a \in \operatorname{Aut}\left(H_{\boldsymbol{a}}\right), c \in H_{\boldsymbol{a}}$, $p= \pm 1$, и $\varepsilon$ действует на $Y$ следующим образом: $\varepsilon(r, n)=(a r+c n, p n)$, $r \in H_{\boldsymbol{a}}, n \in \mathbf{Z}$. Тогда сопряженный автоморфизм $\delta=\widetilde{\varepsilon} \in \operatorname{Aut}(X)$ имеет вид: $\delta(g, z)=\left(\widetilde{a} g,(g, c) z^{p}\right), g \in \Sigma_{\boldsymbol{a}}, z \in \mathbf{T}$.

Обозначим через $\iota$ естественное вложение $\iota: Y \mapsto \mathbf{R} \times \mathbf{Z}, \iota(r, n)=$ $(r, n)$. Пусть $\tau=\widetilde{\iota}$ - сопряженный гомоморфизм $\tau: \mathbf{R} \times \mathbf{T} \mapsto X$. Положим $g_{t}=\tau(t, 1), g_{t} \in \Sigma_{\boldsymbol{a}}$. Тогда $\tau(t, z)=\left(g_{t}, z\right)$. Поскольку, очевидно, $\overline{\iota(Y)}=$ $\mathbf{R} \times \mathbf{Z}$, то $\tau$ - мономорфизм $[24, \S 24.41]$.

Лемма 9 (см. [15]). Пусть а-рачиональное число, $\tau: \mathbf{R} \times \mathbf{T} \mapsto$ $X-$ гомоморфизм, определенный выше. Пусть $K=\left\{\left(t, e^{i t a}\right): t \in \mathbf{R}\right\}-$ подгруппа в $\mathbf{R} \times \mathbf{T}$. Тогда $\overline{\tau(K)} \cong \Sigma_{\boldsymbol{a}}$.

Лемма 10. Пусть $X=\Sigma_{\boldsymbol{a}} \times \mathbf{T}, \alpha_{i j} \in \operatorname{Aut}(X), i, j=1,2,3$. Пусть $\xi_{j}, j=1,2,3$, - независимье случайнье величинь со значениями в 
группе $X$ и с распределениями $\mu_{j}$ такими, что их характеристические функиии не обращаются в нуль. Предположим, что линейнье статистики $L_{1}=\alpha_{11} \xi_{1}+\alpha_{12} \xi_{2}+\alpha_{13} \xi_{3}, L_{2}=\alpha_{21} \xi_{1}+\alpha_{22} \xi_{2}+\alpha_{23} \xi_{3}$, $L_{3}=\alpha_{31} \xi_{1}+\alpha_{32} \xi_{2}+\alpha_{33} \xi_{3}$ независимьл. Тогда распределения $\mu_{j}$ можно так заменить их сдвигами $\mu_{j}^{\prime}$, что распределения $\mu_{j}^{\prime}$ сосредоточень на nodzpynne $\tau(\mathbf{R} \times \mathbf{T})$.

Доказательство этой леммы проводится совершенно аналогично тому, как в [15] доказано соответствующее утверждение для двух независимых случайных величин $\xi_{1}$ и $\xi_{2}$ со значениями в группе $X$ и двух независимых линейных статистик $L_{1}=\xi_{1}+\xi_{2}$ и $L_{2}=\xi_{1}+\delta \xi_{2}$, где $\delta \in \operatorname{Aut}(X)$.

Теорема 2. Пусть $X=\Sigma_{\boldsymbol{a}} \times \mathbf{T}, \alpha_{i j} \in \operatorname{Aut}(X), i, j=1,2,3$. Пусть $\xi_{j}, j=1,2,3$, - независимье случайнье величины со значениями в группе $X$ и с распределениями $\mu_{j}$ такими, что их характеристические функиии не обращаются в нуль. Предположим, что линейнье статистики $L_{1}=\alpha_{11} \xi_{1}+\alpha_{12} \xi_{2}+\alpha_{13} \xi_{3}, L_{2}=\alpha_{21} \xi_{1}+\alpha_{22} \xi_{2}+\alpha_{23} \xi_{3}$, $L_{3}=\alpha_{31} \xi_{1}+\alpha_{32} \xi_{2}+\alpha_{33} \xi_{3}$ независимьл. Тогда либо $\mu_{j}-$ вьгрожденнье распределения, либо $\mu_{j}$ - гауссовские распределения, носители которьх - классьи смежности группьл $X$ относительно подгруппьи, топологически изоморфной $\Sigma_{\boldsymbol{a}}$.

Д о к а з а т е л ь с т в о. Пусть $\tau$ - определенный выше гомоморфизм. Обозначим $G=\tau(\mathbf{R} \times \mathbf{T})$. Как легко видеть, подгруппа $G$ инвариантна относительно любого автоморфизма $\alpha \in \operatorname{Aut}(X)$. По автоморфизму $\alpha$ определим отображение $\bar{\alpha}: \mathbf{R} \times \mathbf{T} \mapsto \mathbf{R} \times \mathbf{T}$ формулой $\bar{\alpha}(t, z)=\tau^{-1} \alpha \tau(t, z)$. Легко проверить, что $\bar{\alpha} \in \operatorname{Aut}(\mathbf{R} \times \mathbf{T})$ и автоморфизму $\bar{\alpha}$ соответствует та же самая матрица, что и автоморфизму $\alpha$. Учитывая лемму 10, можно с самого начала считать, что распределения $\mu_{j}$ сосредоточены на подгруппе $G$. Заметим, что поскольку отображение $\tau$ непрерывно и взаимно однозначно, то по теореме Суслина образы борелевских множеств при отображении $\tau$ - борелевские.

Пусть $\xi$ - случайная величина со значениями в группе $X$, pacпределение которой сосредоточено на подгруппе $G$. Положим $\widetilde{\xi}=\tau^{-1} \xi$. Тогда $\widetilde{\xi}$ - случайная величина со значениями в группе $\underset{\mathbf{R}}{\mathbf{R}} \times \mathbf{T}$. Pacсмотрим случайные величины $\widetilde{\xi}_{j}$. Так как $\tau^{-1} \alpha_{i j} \xi_{j}=\bar{\alpha}_{i j} \widetilde{\xi}_{j}$, то линейные статистики $\widetilde{L}_{1}=\bar{\alpha}_{11} \widetilde{\xi}_{1}+\bar{\alpha}_{12} \widetilde{\xi}_{2}+\bar{\alpha}_{13} \widetilde{\xi}_{3}, \widetilde{L}_{2}=\bar{\alpha}_{21} \widetilde{\xi}_{1}+\bar{\alpha}_{22} \widetilde{\xi}_{2}+\bar{\alpha}_{23} \widetilde{\xi}_{3}$, $\widetilde{L}_{3}=\bar{\alpha}_{31} \widetilde{\xi}_{1}+\bar{\alpha}_{32} \widetilde{\xi}_{2}+\bar{\alpha}_{33} \widetilde{\xi}_{3}$ независимы. Пусть $\widetilde{\mu}_{j}-$ распределения случайных величин $\widetilde{\xi}_{j}$. Поскольку $\mu_{j}=\tau\left(\widetilde{\mu}_{j}\right)$, то утверждение теоремы вытекает из теоремы 1 и леммы 9. Теорема доказана.

\section{СПИСОК ЛИТЕРАТУРЫ}

1. Скитович В. П. Об одном свойстве нормального распределения. - Докл. АН CCCP, 1953, т. 89, с. 217-219. 
2. Darmois G. Analyse générale des liaisons stochastiques. Étude particulière de l'analyse factorielle linéaire. - Rev. Inst. Intern. Statist., 1953, v. 21, p. 2-8.

3. Каган А.М., Линник Ю. В., Рао С. Р. Характеризационные задачи математической статистики. М.: Наука, 1972, 656 с.

4. Фельдман Г. М. Характеризация гауссовского распределения на группах независимостью линейных статистик. - Сиб. матем. журн., 1990, т. 31, № 2, с. 180-190.

5. Фельдман Г. М. К теореме Скитовича-Дармуа на абелевых группах. - Теория вероятн. и ее примен., 1992 , т. 37 , в. 4, с. 695-708.

6. Фельдман Г. М. Теорема Скитовича-Дармуа для компактных групп. - Теория вероятн. и ее примен., 1996, т. 41, в. 4, с. 901-906.

7. Фельдман Г. М. Теорема Скитовича-Дармуа для дискретных периодических абелевых групп. - Теория вероятн. и ее примен., 1997, т. 42, в. 4, с. 747-756.

8. Neuenschwander D., Schott R. The Bernstein and Skitovič-Darmois characterization theorems for Gaussian distributions on groups, symmetric spaces, and quantum groups. - Expo. Math., 1997, v. 15, № 4, p. 289-314.

9. Фельдман Г. М. К теореме Скитовича-Дармуа для конечных абелевых групп. Теория вероятн. и ее примен., 2000, т. 45, в. 3, с. 603-607.

10. Feldman G. M., Graczyk P. On the Skitovich-Darmois theorem for compact Abelian groups. - J. Theoret. Probab., 2000, v. 13, № 3, p. 859-869.

11. Feldman G. M. On a characterization theorem for locally compact Abelian groups. Probab. Theory Related Fields, 2005, v. 133, № 1, p. 345-357.

12. Миронюк M. В., Фельдман Г. М. Независимые линейные статистики на двумерном торе. - Теория вероятн. и ее примен., 2007, т. 52, в. 1, с. 3-20.

13. Фельдман Г. М. Независимые линейные статистики на $a$-адических соленоидах. - Теория вероятн. и ее примен., 2009, т. 54, в. 3, с. 515-532.

14. Feldman G.M., Graczyk P. The Skitovich-Darmois theorem for locally compact Abelian groups. - J. Aust. Math. Soc., 2010, v. 88, № 3, p. 339-352.

15. Feldman G. M., Myronyuk M. V. Independent linear forms on connected Abelian groups. - Math. Nachr., 2011, v. 284, № 2-3, p. 255-265.

16. Feldman G.M. Functional Equations and Characterization Problems on Locally Compact Abelian Groups. Zürich: European Math. Soc., 2008, 256 p. (EMS Tracts in Math., v. 5.)

17. Фельдман Г. М. Гауссовские распределения в смысле Бернштейна на группах. Теория вероятн. и ее примен., 1986, т. 31, в. 1, с. 47-58.

18. Baryshnikov $Y$., Eisenberg B., Stadje $W$. Independent variables with independent sum and difference: $\mathrm{S}^{1}$-case. - J. Multivariate Anal., 1993, v. 45, № 2, p. 161-170.

19. Parthasarathy K. R., Ranga Rao R., Varadhan S.R.S. Probability distributions on locally compact abelian groups. - Illinois J. Math., 1963, v. 7, p. 337-369.

20. Parthasarathy K.R. Probability measures on metric spaces. New York-London: Academic Press, 1967, 276 p.

21. Фельдман Г. М. О разложении гауссовского распределения на группах. - Теория вероятн. и ее примен., 1977, т. 22 , в. 1, с. 136-143.

22. Fel'dman G.M. Arithmetic of Probability Distributions, and Characterization Problems on Abelian Groups. Providence: Amer. Math. Soc., 1993, 223 p. (Transl. Math. Monogr., v. 116.)

23. Djoković D. Ž. A representation theorem for $\left(X_{1}-1\right)\left(X_{2}-1\right) \cdots\left(X_{n}-1\right)$ and its applications. - Ann. Polon. Math., 1969, v. 22, p. 189-198.

24. Хьюитm Э., Росс К. Абстрактный гармонический анализ, т. 1. М.: Наука, 1975, $656 \mathrm{c}$

Поступила в редакцию

14.III.2013

Исправленный вариант 28.IV.2014 